\title{
QUASIINVARIANT GAUSSIAN MEASURES FOR ONE-DIMENSIONAL HAMILTONIAN PARTIAL DIFFERENTIAL EQUATIONS
}

\author{
NIKOLAY TZVETKOV \\ Université de Cergy-Pontoise, Cergy-Pontoise, F-95000, UMR 8088 du CNRS, France; \\ email: nikolay.tzvetkov@u-cergy.fr
}

Received 17 June 2015; accepted 20 October 2015

\begin{abstract}
We prove the quasiinvariance of Gaussian measures (supported by functions of increasing Sobolev regularity) under the flow of one-dimensional Hamiltonian partial differential equations such as the regularized long wave, also known as the Benjamin-Bona-Mahony (BBM) equation.
\end{abstract}

2010 Mathematics Subject Classification: 35Q53

\section{Introduction}

1.1. Motivation. Our motivation for this work is twofold. On the one hand, there is an extensive literature about the transport of Gaussian measures under nonlinear transformations (see, for example, $[4,5,18,19,32])$. These works treat either general nonlinear transformations close to the identity (see, for example, [32]) or transformations generated by vector fields, under an exponential integrability assumption (see, for example, [19]). It was however not clarified how much these results apply in the context of Hamiltonian partial differential equations (PDEs).

On the other hand, there is an extensive literature about invariant Gaussian type measures (absolutely continuous with respect to Gaussian measures) under the flows of Hamiltonian PDEs (see, for example, [7-11, 13, 15, 16, 20, 21, 23, 26, 27, $29,30,33,35-38,40])$. In most cases the support of the measure consists of lowregularity functions. Two exceptions are the Korteweg-de Vries (KdV) and the

(c) The Author 2015. This is an Open Access article, distributed under the terms of the Creative Commons Attribution licence (http://creativecommons.org/licenses/by/4.0/), which permits unrestricted re-use, distribution, and reproduction in any medium, provided the original work is properly cited. 
Benjamin-Ono equations, where one can use the high-order (that is, controlling high-order Sobolev norms) conservation laws in order to get invariant Gaussian type measures supported by fairly smooth functions (see $[36,37,40]$ ).

In recent work $[27,28,34]$ on invariant Gibbs measures for the derivative nonlinear Schrödinger equation, both the above aspects were involved. Namely, the analysis of [27] combined with the remarkable description of the transport of the Brownian loop under gauge transformations performed in [28] led to the proof of the invariance of the weighted Wiener measure constructed in [34].

The existence of conservation laws controlling higher Sobolev norms is an exceptional event. Therefore, for Hamiltonian PDEs where conservation laws of high order are not available, it is not clear how the transport of Gaussian measures, supported by functions of high Sobolev regularity, behaves under the corresponding Hamiltonian flow. Our goal here is to make a progress in this direction. Namely, we will show that, in the case of regularized long wave equations, Gaussian measures supported by functions of arbitrary high Sobolev regularities are quasiinvariant by the flow of the corresponding equations. We recall that a measure $\mu$ on a space $X$ is called quasiinvariant under a transformation $\Phi: X \rightarrow X$ if its image under $\Phi$ is absolutely continuous with respect to $\mu$.

The existence of a quasiinvariant measure in the context of a Hamiltonian PDE represents a step in the macroscopic description of the corresponding flow: in particular, a set of a positive measure is always transported to a set of a positive measure. If in addition the quasiinvariance is proved together with good quantitative bounds, then it may have consequences concerning the long-time behaviour of the solutions (see, for example, Remark 7.4 below).

\subsection{Derivation of a Benjamin-Bona-Mahony (BBM) type model. If} one considers shallow small-amplitude long water waves, one obtains that the evolution of the water surfaces $u$ satisfies (formally) the equation

$$
\partial_{t} u+\partial_{x} u+\varepsilon_{1} \partial_{x}^{3} u+\varepsilon_{2} \partial_{x}\left(u^{2}\right)=O\left(\varepsilon_{1}^{2}+\varepsilon_{2}^{2}\right)
$$

In (1.1), $\varepsilon_{1}$ represents the square of the ratio between the depth of the fluid and the typical wavelength while $\varepsilon_{2}$ represents the ratio between the wave amplitude and the depth (see, for example, [25]). Both $\varepsilon_{1}$ and $\varepsilon_{2}$ are small parameters. In the regime $\varepsilon_{1} \approx \varepsilon_{2}$, one takes into account both linear and nonlinear effects. Therefore by neglecting the error in the right-hand side of (1.1) one ends up with the famous $\mathrm{KdV}$ equation. The $\mathrm{KdV}$ equation has a highly oscillating linear part and a nonlinear part with a strong effect (derivative loss). Since at first order 
$\partial_{t} u \approx-\partial_{x} u$, in [3] the authors introduced the model

$$
\partial_{t} u+\partial_{x} u-\partial_{t} \partial_{x}^{2} u+\partial_{x}\left(u^{2}\right)=0
$$

as an alternative of the $\mathrm{KdV}$ model (in (1.2), we dropped the $\varepsilon_{1,2}$ dependence). If we write (1.2) in the form

$$
\partial_{t} u+\left(1-\partial_{x}^{2}\right)^{-1} \partial_{x} u+\left(1-\partial_{x}^{2}\right)^{-1} \partial_{x}\left(u^{2}\right)=0,
$$

we observe that the model (1.2) has a slowly oscillating linear part coupled with a weak nonlinearity (with smoothing of degree one). Despite this difference with respect to the $\mathrm{KdV}$ model, (1.2) is supposed to describe a similar balance between linear and nonlinear effects.

One may naturally consider the following generalization (generalized dispersion) of the $\mathrm{KdV}$ equation:

$$
\partial_{t} u+\partial_{x} u-\left|D_{x}\right|^{\gamma} \partial_{x} u+\partial_{x}\left(u^{2}\right)=0 .
$$

For $\gamma=2$, we recover the KdV model, but for $\gamma=1$ one gets the Benjamin-Ono equation, which is a model that can be derived similarly to the $\mathrm{KdV}$ model, but in the context of internal waves.

Following the same argument as for deriving (1.2), we end up with the following generalization of (1.2):

$$
\partial_{t} u+\partial_{t}\left|D_{x}\right|^{\gamma} u+\partial_{x} u+\partial_{x}\left(u^{2}\right)=0 .
$$

For $\gamma=2$ we recover (1.2), while for $\gamma=1$ we deal with a Benjamin-Ono type model. The goal of this work is to study (1.4) with initial data distributed by Gaussian measures in Sobolev spaces of an arbitrary regularity. Observe that, at least formally, if we multiply (1.4) by $u$ and integrate in $x$, we obtain that a Sobolev type norm of order $\gamma / 2$ of $u$ is conserved by (1.4). This global information is the only useful a priori bound for (1.4) we are aware of, and it will play an important role in the analysis below. In fact, one does not expect to have a priori bounds controlling higher Sobolev norms in the context of (1.4). Indeed, in the case when $\gamma=2$ it is shown in [31] that (1.2) has three conservation laws (of order 0,0 , and 1 , respectively) but no higher-order laws, in contrast to completely integrable equations such as the KdV equation and the BenjaminOno equation. As a consequence there are only a limited number of Gibbs type measures available for the BBM equation (1.2). Therefore, at least for $\gamma=2$, our results concerning the existence of quasiinvariant Gaussian measures for (1.4) cannot be obtained by the approach of $[36,37,40]$ exploiting the existence of Gibbs type measures associated with higher-order conservation laws. 
1.3. Statement of the results. We consider (1.4), posed on the onedimensional torus. Since the $x$ mean value is preserved by (1.4), we shall consider (1.4) as a dynamical system on the Sobolev spaces of zero $x$ mean value functions (equivalently functions having vanishing zero Fourier coefficient). We denote by $H^{s}$ the Sobolev space of zero mean functions (see the notation section below for a precise definition). The next statement shows that (1.4) defines a dynamical system on $H^{s}, s \geqslant \gamma / 2$.

Proposition 1.1. Let $\gamma>1$, and let $\sigma \geqslant \gamma / 2$. Then for every $u_{0} \in H^{\sigma}$ there is a unique global solution of (1.4) in $C\left(\mathbb{R} ; H^{\sigma}\right)$. Moreover, if we denote by $\Phi(t)$ the flow of (1.4), then, for every $t \in \mathbb{R}, \Phi(t)$ is a continuous bijection on $H^{\sigma}$.

Once this result is established one may naturally ask qualitative questions of the global behaviour of $(\Phi(t))_{t \in \mathbb{R}}$ as a dynamical system on $H^{\sigma}$. As already mentioned, in this work we will study the transport of some Gaussian measures by $\Phi(t)$ (for $\sigma$ not necessarily small).

We next introduce these measures. Let $s \geqslant 1$ be an integer. Denote by $\mu_{s}$ the Gaussian measure induced by the random Fourier series

$$
\varphi_{s}(\omega, x)=\sum_{n \neq 0} \frac{g_{n}(\omega)}{|n|^{s+\gamma / 2}} e^{i n x},
$$

where $g_{n}=\overline{g_{-n}}$ and $\left(g_{n}\right)_{n>0}$ is a system of independent and identically distributed standard complex Gaussians; that is,

$$
g_{n}=\frac{1}{\sqrt{2}}\left(h_{n}+i l_{n}\right),
$$

where $h_{n}, l_{n} \in \mathcal{N}(0,1)$ are independent. Strictly speaking, the measure $\mu_{s}$ also depends on $\gamma$, but we do not make this dependence explicit. For $\gamma>1$, the measure $\mu_{s}$ can be seen as a Gaussian measure on $H^{s}$. Therefore, thanks to Proposition 1.1 , for every $\gamma>1$ the flow $\Phi(t)$ is defined to be $\mu_{s}$ almost surely, provided $s \geqslant \gamma / 2$. Our main goal is to prove the following statement.

THEOREM 1.2. Let $\gamma>\frac{4}{3}$. Then for every integer $s \geqslant \gamma / 2$ the measure $\mu_{s}$ is quasiinvariant by the flow $\Phi(t)$, for every $t \in \mathbb{R}$.

We also obtain some quantitative bounds on the densities of the transported measures (see, for example, Lemma 8.1 below). However, the present information these bounds give on the densities at time $t$ seems quite weak to be useful for giving new long-time bounds on the solutions a.s. with respect to $\mu_{s}$ (see, for example, Remark 7.4 below). It would be interesting to improve on these bounds. 
For that reason, we decided to keep the quantitative part of our argument, hoping that it may be of some interest in eventual further developments.

We did not try to optimize the restriction $\gamma>\frac{4}{3}$, our goal being to achieve a value of $\gamma$ smaller than $\frac{3}{2}$ which allows us to go beyond the Cameron-Martin threshold (see also Proposition 1.3 below). The assumption that $s$ is an integer is not essential, and most probably can be removed.

The measure $\mu_{s}$ can be seen as a normalized version of the formal object

$$
\exp \left(-\|u\|_{H^{s+\gamma / 2}}^{2}\right) d u .
$$

For $\gamma>1$, the triple (Id, $H^{s+\gamma / 2}, H^{s}$ ) forms a Wiener space, and $\mu_{s}$ is the standard Gaussian measure on $H^{s}$ with variance parameter 1 . The space $H^{s+\gamma / 2}$ is the canonical Hilbert space (the so-called Cameron-Martin space) in this construction, but the space $H^{s}$ may be replaced by any $H^{\sigma}$ with $\sigma<s+\gamma / 2-1 / 2$.

In the case when $s=0$, thanks to the conservation of the Sobolev norm of order $\gamma / 2$ one can get the invariance of the measure $\mu_{0}$, by employing the wellestablished methods of invariance of Gibbs measures, at least for $\gamma \geqslant 2$ (see [22] for the case when $\gamma=2$ ). The extension to some values of $\gamma<2$ would require some elaborations on the local-in-time analysis in the proof of Proposition 1.1 (to obtain the existence of the dynamics, locally in time, on the support of the measure $\mu_{0}$ ).

1.4. Comparison with Cameron-Martin type of results. It is instructive to compare the result of Theorem 1.2 with the Cameron-Martin theorem [17] and a result by Ramer [32]. Denote by $S(t)$ the free evolution associated to (1.4); that is,

$$
S(t)=\exp \left(-t\left(1+\left|D_{x}\right|^{\gamma}\right)^{-1} \partial_{x}\right) .
$$

Then, thanks to the Duhamel formula, the map $\Phi(t)$ can be written as

$$
\Phi(t)\left(u_{0}\right)=S(t)\left(u_{0}\right)+\left((\gamma-1) \text { smoother part depending on } u_{0}\right) .
$$

The free evolution $S(t)$ preserves the Sobolev regularity and the measure $\mu_{s}$ (thanks to the invariance of the Gaussians by rotations). Therefore the CameronMartin theorem implies that $\mu_{s}$ is quasiinvariant by maps of the form

$$
S(t)\left(u_{0}\right)+\left(\left(\frac{1}{2}+\varepsilon\right) \text { smoother part independent of } u_{0}\right),
$$

for some $\varepsilon>0$. Therefore in the range $\gamma \in\left(\frac{4}{3}, \frac{3}{2}\right]$ the result of Theorem 1.2 goes beyond the naive intuition dictated by the Cameron-Martin theorem. We find this phenomenon interesting (see also Proposition 1.3 below). 
Let us next compare the result of Theorem 1.2 with a result by Ramer. Using once again the above-mentioned properties of $S(t)$, one gets that the result of Ramer implies that $\mu_{s}$ is quasiinvariant by maps of the form

$$
S(t)\left(u_{0}\right)+\left((1+\varepsilon) \text { smoother part which may depend on } u_{0}\right),
$$

for some $\varepsilon>0$. Therefore for $\gamma>2$ the result of Theorem 1.2 follows from the work by Ramer [32]; that is, the BBM model [3] is the border line (but not covered by [32]). The assumption that $\gamma>2$ is done in order to ensure the Hilbert-Schmidt property of the perturbation imposed in [32] (see Section 3 below). The work of Ramer deals with general maps, and in this setting the $1+\varepsilon$ regularization condition (in dimension one) looks optimal. The reason why Theorem 1.2 goes beyond the result of [32] is that we deal with very particular nonlinear maps induced by Hamiltonian flows. Here our work is close in spirit to the articles by Cruzeiro $[18,19]$, which prove abstract results concerning the existence of quasiinvariant measures under the flows of (not necessarily smooth) vector fields. In the work of Cruzeiro, the existence of the dynamics (the analogue of Proposition 1.1) is already a nontrivial issue (see also [1, 5] for more recent works). Concerning the quasiinvariance statement in [19], it is done under an exponential integrability assumption of the divergence of the corresponding vector field. One may wish to see the result of Theorem 1.2 as an instance where such an integrability condition is checked 'in practice'.

We end the discussion about the comparison between Theorem 1.2 and the Cameron-Martin type of results by the following statement.

Proposition 1.3. Let $\gamma \in\left(\frac{4}{3}, \frac{3}{2}\right)$. Consider the linear PDE

$$
\partial_{t} u+\partial_{t}\left|D_{x}\right|^{\gamma} u+\partial_{x} u+\partial_{x}(h)=0,
$$

where $h \in H^{\sigma}$ (independent of $t$ ) for some $\sigma<s+\gamma / 2-1 / 2$ is fixed. Suppose that $h \notin H^{s+\gamma / 2-1 / 2}$. Denote by $\Sigma(t)$ the (well-defined) flow of (1.5). Then for $t \neq 0$ the transport of $\mu_{s}$ by $\Sigma(t)$ is a measure that is singular with respect to $\mu_{s}$.

In Proposition 1.3, the fixed function $h$ is supposed to have the typical regularity on the support of $\mu_{s}$. In other words, if $u$ is a solution of (1.4) with data on the support of $\mu_{s}$, then we take $h$ with the regularity of $u^{2}$ obtained by the deterministic estimates of Section 2 below (and not more). Therefore, for $\gamma \in\left(\frac{4}{3}, \frac{3}{2}\right)$, the result of Theorem 1.2 seems to go beyond a Cameron-Martin type result, and it relies on a 'regularization property' of the flow associated with (1.4).

1.5. Organization of the paper. The remaining part of this paper is organized as follows. We complete this introduction by introducing some notation. 
In Section 2, we prove the existence of the dynamics and some useful approximation properties. In Section 3 we obtain the result of Theorem 1.2 for $\gamma>2$ as a consequence of [32]. Next, in Section 4, we establish a useful infinite-dimensional change of variables formula. In Section 5, we get the suitable for our purposes (deterministic) energy estimate. In Section 6, we establish the averaging with respect to $\mu_{s}$ properties, needed for our analysis. In Section 7, we establish the measure evolution property by an argument in the spirit of the proof of the global regularity for the two-dimensional Euler equation. In Section 8 , we complete the proof of the main result by some basic measure theory considerations. Finally, in Section 9, we prove Proposition 1.3 as a simple consequence of the Cameron-Martin argument.

1.6. Notation. If a real-valued $f$ is given by its Fourier expansion

$$
f(x)=\sum_{n \in \mathbb{Z}} \hat{f}(n) e^{i n x}, \quad \hat{f}(n)=\overline{\hat{f}(-n)},
$$

for $s \in \mathbb{R}$, we define its Sobolev norm as

$$
\|f\|_{\mathcal{H}^{s}}=\|f\|_{s}=\left(\sum_{n \in \mathbb{Z}}\langle n\rangle^{2 s}|\hat{f}(n)|^{2}\right)^{1 / 2},
$$

where $\langle n\rangle=1+|n|$. We denote by $\mathcal{H}^{s}$ the space of real-valued $f$ such that (1.6) is finite. It is well known that $\mathcal{H}^{s}$ is a Hilbert space (with the natural scalar product). We denote by $H^{s}$ the closed subspace of $\mathcal{H}^{s}$ of functions with zero Fourier coefficient; that is,

$$
H^{s}=\left\{f \in \mathcal{H}^{s}: \hat{f}(0)=0\right\} .
$$

Since the mean value is preserved by (1.4), we have that $H^{s}$ is a natural space for the solutions of (1.4). We consider $H^{s}$, equipped with the norm

$$
\|f\|_{H^{s}}=\frac{1}{\sqrt{2}}\left(\sum_{n \in \mathbb{Z}}|n|^{2 s}|\hat{f}(n)|^{2}\right)^{1 / 2}=\left(\sum_{n=1}^{\infty}|n|^{2 s}|\hat{f}(n)|^{2}\right)^{1 / 2} .
$$

For elements in $H^{s}$, the norms (1.6) and (1.7) are equivalent. We define the Fourier multipliers $\left|D_{x}\right|^{s}$ as

$$
\left|D_{x}\right|^{s}(f)(x)=\sum_{n \in \mathbb{Z}}|n|^{s} \hat{f}(n) e^{i n x}
$$

Then

$$
\|f\|_{H^{s}}=\frac{1}{2 \sqrt{\pi}}\left\|\left|D_{x}\right|^{s} f\right\|_{L^{2}} .
$$


Observe that $\|\cdot\|_{H^{0}}$ differs slightly from $\|\cdot\|_{L^{2}}$ (which 'explains' the appearance of the $4 \pi$ factor in the definition of $\chi_{r}(u)$ below). We denote by $\pi_{N}$ the Dirichlet projector; that is,

$$
\pi_{N}(f)=\sum_{|n| \leqslant N} \hat{f}(n) e^{i n x} .
$$

We denote by $\mu_{s, r}$, the measure defined by

$$
d \mu_{s, r}(u)=\chi_{r}(u) d \mu_{s}(u)
$$

where

$$
\chi_{r}(u)=\chi\left(r^{-1}\left(\|u\|_{L^{2}}^{2}+4 \pi\|u\|_{H^{\gamma / 2}}^{2}\right)\right)
$$

and $\chi: \mathbb{R} \rightarrow \mathbb{R}$ denotes the characteristic function of the set $[0,1]$.

For $s \in \mathbb{R}$ and $R \geqslant 0$, we set $B_{R, s}=\left\{u \in H^{s}:\|u\|_{H^{s}} \leqslant R\right\}$.

\section{Construction and general properties of the flows and the approximated flows}

2.1. Existence of the dynamics. We assume $\gamma>1$ throughout this section. Consider the truncated version of (1.4),

$$
\partial_{t} u+\partial_{t}\left|D_{x}\right|^{\gamma} u+\partial_{x} u+\partial_{x} \pi_{N}\left(\left(\pi_{N} u\right)^{2}\right)=0 .
$$

We consider (2.1), posed on the one-dimensional torus and with (nontruncated) initial data in $H^{s}$.

LEMMA 2.1. Let $\gamma>1$. Then, for every $\sigma \geqslant 0$,

$$
\left\|\left(1+\left|D_{x}\right|^{\gamma}\right)^{-1} \partial_{x}(u v)\right\|_{\sigma} \leqslant C_{\sigma}\left(\|u\|_{\sigma}\|v\|_{\gamma / 2}+\|u\|_{\gamma / 2}\|v\|_{\sigma}\right) .
$$

Proof. Since $\left(1+\left|D_{x}\right|^{\gamma}\right)^{-1} \partial_{x}$ is bounded on $\mathcal{H}^{\sigma}$, the proof of direct consequence of the classical product estimate

$$
\|u v\|_{\sigma} \leqslant C_{\sigma}\left(\|u\|_{\sigma}\|v\|_{L^{\infty}}+\|u\|_{L^{\infty}}\|v\|_{\sigma}\right)
$$

and the Sobolev embedding $\mathcal{H}^{\gamma / 2} \subset L^{\infty}$.

Using Lemma 2.1, one gets the following uniform in $N$ local well-posedness result for (2.1).

LEMMA 2.2. Let $\sigma \geqslant \gamma / 2$. Then for every $u(0) \in H^{\sigma}$ there is a time $\tau>0$ depending only on $\|u(0)\|_{H^{\gamma / 2}}$ and there is a unique solution of (2.1) in $C([-\tau$, $\left.\tau] ; H^{\sigma}\right)$ with initial data $u(0)$. Moreover, $\|u\|_{L^{\infty}\left([-\tau, \tau] ; H^{\sigma}\right)} \leqslant 2\|u(0)\|_{H^{\sigma}}$. 
Proof. We proceed by a fixed point argument. One may rewrite (2.1) as the integral equation

$$
u(t)=S(t)(u(0))-\int_{0}^{t} S(t-\tau)\left(\left(1+\left|D_{x}\right|^{\gamma}\right)^{-1} \partial_{x} \pi_{N}\left(\left(\pi_{N} u(\tau)\right)^{2}\right)\right) d \tau .
$$

Using Lemma 2.1, one can look for a fixed point of the map $F_{u(0)}(u)$, defined by the right-hand side of (2.3) in a suitable ball of the space $C\left([-\tau, \tau] ; H^{\gamma / 2}\right)$, where $\tau=c\left(1+\|u(0)\|_{H^{\gamma / 2}}\right)^{-1}$ and $c$ is a small constant. Indeed, using Lemma 2.1 and the uniform bounds for $\pi_{N}$ on $H^{\gamma / 2}$, we can obtain that, for $u \in C\left([-\tau, \tau] ; H^{\gamma / 2}\right)$,

$$
\left\|F_{u(0)}(u)\right\|_{L^{\infty}\left([-\tau, \tau] ; H^{\gamma / 2}\right)} \leqslant\left\|u_{0}\right\|_{H^{\gamma / 2}}+C \tau\|u\|_{L^{\infty}\left([-\tau, \tau] ; H^{\gamma / 2}\right)}^{2} .
$$

Therefore, the space $E$ defined by

$$
E \equiv\left\{u \in C\left([-\tau, \tau] ; H^{\gamma / 2}\right):\|u\|_{L^{\infty}\left([-\tau, \tau] ; H^{\gamma / 2}\right)} \leqslant 2\|u(0)\|_{H^{\gamma / 2}}\right\}
$$

is such that $F_{u(0)}(E) \subset E$, provided the constant $c$ in the definition of $\tau$ is small enough. By invoking once again Lemma 2.1 and the uniform bounds for $\pi_{N}$ on $H^{\gamma / 2}$, we can obtain that, for $u, v \in E$,

$$
\left\|F_{u(0)}(u)-F_{u(0)}(v)\right\|_{L^{\infty}\left([-\tau, \tau] ; H^{\gamma / 2}\right)} \leqslant \frac{1}{2}\|u-v\|_{L^{\infty}\left([-\tau, \tau] ; H^{\gamma / 2}\right)},
$$

by possibly taking an even smaller value of the constant $c$ involved in the definition of $\tau$. Therefore $F_{u(0)}$ is a contraction on $E$. The fixed point of this contraction provides the solution of (2.1) we are looking for.

Let us now turn the propagation the $H^{\sigma}$-regularity of the obtained solution $u$. This regularity is preserved for very small times of order $\left(1+\|u(0)\|_{H^{\sigma}}\right)^{-1}$ by the fixed-point argument that we have just presented. In order to show that the regularity is preserved for longer times, we use Lemma 2.1 and the uniform bounds for $\pi_{N}$ on $H^{\sigma}$ in order to obtain that

$$
\|u\|_{L^{\infty}\left([-\tau, \tau] ; H^{\sigma}\right)} \leqslant\left\|u_{0}\right\|_{H^{\sigma}}+C_{\sigma} \tau\|u\|_{L^{\infty}\left([-\tau, \tau] ; H^{\sigma}\right)}\|u\|_{L^{\infty}\left([-\tau, \tau] ; H^{\gamma / 2}\right)} .
$$

Therefore the $H^{\sigma}$-regularity is preserved for time $\tau=c\left(1+\|u(0)\|_{H^{\gamma / 2}}\right)^{-1}$, where the constant $c$ is sufficiently small, depending only on $\sigma$.

The uniqueness statement follows by using that, if $u_{1}$ and $u_{2}$ are two solutions of (2.1) in $C\left([-\tau, \tau] ; H^{\sigma}\right)$, then, by using Lemma 2.1 and the uniform bounds for $\pi_{N}$ on $H^{\sigma}$, we can obtain that, for any interval $\left[-\tau_{1}, \tau_{1}\right], \tau_{1} \leqslant \tau$,

$$
\left\|u_{1}-u_{2}\right\|_{L^{\infty}\left(\left[-\tau_{1}, \tau_{1}\right] ; H^{\sigma}\right)} \leqslant C_{\sigma} \tau_{1}\left\|u_{1}-u_{2}\right\|_{L^{\infty}\left(\left[-\tau_{1}, \tau_{1}\right] ; H^{\sigma}\right)}\left\|u_{1}+u_{2}\right\|_{L^{\infty}\left([-\tau, \tau] ; H^{\sigma}\right)} .
$$

We therefore conclude that $u_{1}=u_{2}$ on $\left[-\tau_{1}, \tau_{1}\right]$, where $\tau_{1}$ is such that

$$
\tau_{1} C_{\sigma}\left(\left\|u_{1}\right\|_{L^{\infty}\left([-\tau, \tau] ; H^{\sigma}\right)}+\left\|u_{2}\right\|_{L^{\infty}\left([-\tau, \tau] ; H^{\sigma}\right)}\right)<\frac{1}{2} .
$$


Then we cover $[-\tau, \tau]$ by intervals of size $\tau_{1}$, and we repeat the previous reasoning to conclude that $u_{1}=u_{2}$ on $[-\tau, \tau]$. The continuity statements are consequences of the previous analysis. This completes the proof of Lemma 2.2.

By the invariance of (1.4) with respect to time translations, the statement of Lemma 2.2 holds true with initial data given at any time $t_{0} \in \mathbb{R}$ on the interval $\left[t_{0}-\tau, t_{0}+\tau\right]$, where $\tau$ depends only on $\left\|u\left(t_{0}\right)\right\|_{H^{\gamma / 2}}$.

One can similarly obtain the following local well-posedness result for (1.4).

LEMmA 2.3. Let $\sigma \geqslant \gamma / 2$. Then for every $u(0) \in H^{\sigma}$ there is a time $\tau>0$ depending only on $\|u(0)\|_{H^{\gamma / 2}}$ and there is a unique solution of (1.4) in $C([-\tau$, $\left.\tau] ; H^{\sigma}\right)$ with initial data $u(0)$. Moreover,

$$
\|u\|_{L^{\infty}\left([-\tau, \tau] ; H^{\sigma}\right)} \leqslant 2\|u(0)\|_{H^{\sigma}} .
$$

The next lemma is of key importance.

LEMMA 2.4. Let u be a local solution of (2.1), given by Lemma 2.2. Then

$$
\frac{d}{d t}\left(\|u(t)\|_{L^{2}}^{2}+4 \pi\|u(t)\|_{H^{\gamma / 2}}^{2}\right)=0 .
$$

A similar statement holds for the solutions of (1.4).

Proof. Let $u$ be a local solution of (2.1), given by Lemma 2.2. Then we take the $L^{2}$ scalar product of (2.1) with $u$, and using that

$$
\left(\partial_{x} \pi_{N}\left(\left(\pi_{N} u\right)^{2}\right), u\right)=\frac{1}{3} \int \partial_{x}\left(\left(\pi_{N} u\right)^{3}\right)=0, \quad\left(u, \partial_{x} u\right)=0,
$$

and

$$
\left(\partial_{t} u, u\right)=\frac{1}{2} \partial_{t}\|u\|_{L^{2}}^{2}, \quad\left(\partial_{t}\left|D_{x}\right|^{\gamma} u, u\right)=\frac{1}{2} \partial_{t}\left\|\left|D_{x}\right|^{\gamma / 2} u\right\|_{L^{2}}^{2},
$$

we obtain that (2.5) holds.

Using Lemmas 2.2, 2.3, and the conservation law displayed by Lemma 2.4, one readily gets Proposition 1.1 and also the global well-posedness in $H^{\sigma}$ of (2.1), 'uniformly' in $N$. Let us denote by $\Phi(t)$ and $\Phi_{N}(t)$ the global flows on $H^{\sigma}, \sigma \geqslant \gamma / 2$ of (1.4) and (2.1), respectively. By iterating the local bounds we get the following statement.

Proposition 2.5. Let $\sigma \geqslant \gamma / 2$. For every $R>0$ there is a constant $C$ such that, for every $v \in H^{\sigma}$ such that $\|v\|_{H^{\gamma / 2}} \leqslant R$, and every $N \geqslant 1$, one has the bound

$$
\|\Phi(t)(v)\|_{H^{\sigma}}+\left\|\Phi_{N}(t)(v)\right\|_{H^{\sigma}} \leqslant e^{C(1+|t|)}\|v\|_{H^{\sigma}} .
$$


Proof. Let $u$ be a solution of (2.1). Using (2.5) for every $t$, we can iterate the local bound (2.4) $[|t| / \tau]+1$ times to obtain that

$$
\|u(t)\|_{H^{\sigma}} \leqslant 2^{[|t| / \tau]+1}\|u(0)\|_{H^{\sigma}} .
$$

A similar analysis applies for the solutions of (1.4). This completes the proof of Proposition 2.5.

As a consequence of Proposition 2.5, one also gets the following statement.

Proposition 2.6. Let $\sigma \geqslant \gamma / 2$. Then for every $T>0$ and $R>0$ there exists $R^{\prime}>0$ such that

$$
\bigcup_{N \in \mathbb{N}} \bigcup_{t \in[-T, T]}\left(\Phi_{N}(t)\left(B_{R, \sigma}\right) \cup \Phi(t)\left(B_{R, \sigma}\right)\right) \subset B_{R^{\prime}, \sigma} .
$$

2.2. Approximation properties. We have the following basic approximation property.

Proposition 2.7. Let $\sigma \geqslant \gamma / 2$. Fix $t \in \mathbb{R}, R>0$, and a compact $K \subset B_{R, \sigma}$. Then for every $\varepsilon>0$ there exists $N_{0}$ such that, for every $N \geqslant N_{0}$,

$$
\left\|\Phi(t)(v)-\Phi_{N}(t)(v)\right\|_{H^{\sigma}}<\varepsilon, \quad \forall v \in K .
$$

Proof. Let $u$ and $u_{N}$ be solutions of (1.4) and (2.1) with the same initial data $v \in K$. Then $w_{N} \equiv u-\pi_{N} u_{N}$ solves the equation

$$
\partial_{t} w_{N}+\partial_{t}\left|D_{x}\right|^{\gamma} w_{N}+\partial_{x} w_{N}+\partial_{x}\left(u^{2}-\pi_{N}\left(\left(\pi_{N} u\right)^{2}\right)\right)=0 .
$$

Next, we can write

$$
u^{2}-\pi_{N}\left(\left(\pi_{N} u_{N}\right)^{2}\right)=\left(1-\pi_{N}\right)\left(u^{2}\right)+\pi_{N}\left(w_{N}\left(u+\pi_{N} u_{N}\right)\right) .
$$

By using the estimate of Lemma 2.1 and Proposition 2.6, we obtain that there is a constant $C$ only depending on $\sigma, t$, and $R$ such that

$$
\left\|w_{N}\right\|_{L^{\infty}\left([-\tau, \tau] ; H^{\sigma}\right)} \leqslant C \tau\left\|w_{N}\right\|_{L^{\infty}\left([-\tau, \tau] ; H^{\sigma}\right)}+C\left\|\left(1-\pi_{N}\right)\left(u^{2}\right)\right\|_{L^{\infty}\left([-\tau, \tau] ; H^{\sigma}\right)} .
$$

We are now in a position to use the following lemma.

LEMMA 2.8. For every $\varepsilon>0$ there is $N_{0}$ such that, for every $N \geqslant N_{0}$ and every $v \in K$,

$$
\left\|\left(1-\pi_{N}\right)\left((\Phi(t)(v))^{2}\right)\right\|_{L^{\infty}\left([-\tau, \tau] ; H^{\sigma}\right)}<\varepsilon .
$$


Proof. Let $\varepsilon>0$. Since the map $v \mapsto \Phi(t)(v)$ is continuous from $H^{\sigma}$ to $C\left([-\tau, \tau] ; H^{\sigma}\right)$, we obtain that the image of $K$ under this map is a compact in $C\left([-\tau, \tau] ; H^{\sigma}\right)$. Therefore, using Lemma 2.1 and Proposition 2.6, we obtain that there exists a finite set $J$ such that $\left(\Phi(t)\left(v_{j}\right)\right)_{j \in J}$ has the property

$$
\forall v \in K, \quad \exists j \in J, \quad\left\|(\Phi(t)(v))^{2}-\left(\Phi(t)\left(v_{j}\right)\right)^{2}\right\|_{L^{\infty}\left([-\tau, \tau] ; H^{\sigma}\right)}<\frac{\varepsilon}{2} .
$$

Therefore, it remains to show that, for every $j$ in the finite set $J$, there is $N_{0}$ such that, for every $N \geqslant N_{0}$,

$$
\left\|\left(1-\pi_{N}\right)\left(\left(\Phi(t)\left(v_{j}\right)\right)^{2}\right)\right\|_{L^{\infty}\left([-\tau, \tau] ; H^{\sigma}\right)}<\frac{\varepsilon}{2} .
$$

Now, thanks to the (uniform) continuity of the map $t \mapsto\left(\Phi(t)\left(v_{j}\right)\right)^{2}$ from $[-\tau, \tau]$ to $H^{\sigma}$, we obtain that there is a finite set $\left(t_{l}\right)_{l \in \Lambda}$ of $[-\tau, \tau]$ such that

$$
\forall t \in[-\tau, \tau], \quad \exists l \in \Lambda, \quad\left\|\left(\Phi(t)\left(v_{j}\right)\right)^{2}-\left(\Phi\left(t_{l}\right)\left(v_{j}\right)\right)^{2}\right\|_{H^{\sigma}}<\frac{\varepsilon}{4} .
$$

We are therefore reduced to showing that, for every $j \in J$ and every $l \in \Lambda$, there is $N_{0}$ such that, for every $N \geqslant N_{0}$,

$$
\left\|\left(1-\pi_{N}\right)\left(\left(\Phi\left(t_{l}\right)\left(v_{j}\right)\right)^{2}\right)\right\|_{H^{\sigma}}<\frac{\varepsilon}{4} .
$$

The last statement is a direct consequence of the definition of the Sobolev space $H^{\sigma}$. This completes the proof of Lemma 2.8.

Using Lemma 2.8, we obtain that, if the constant $C$ involved in (2.7) satisfies $C \tau<\frac{1}{2}$, then

$$
\left\|w_{N}\right\|_{L^{\infty}\left([-\tau, \tau] ; H^{\sigma}\right)} \leqslant \Lambda(N, v) .
$$

Here and in the remainder of the proof we denote by $\Lambda(N, v)$ a generic quantity such that, for every $\varepsilon>0$, there is $N_{0}$ such that, for every $N \geqslant N_{0}$ and every $v \in K, \Lambda(N, v)<\varepsilon$.

Next, we can perform the same analysis to arrive at the bound

$$
\left\|w_{N}\right\|_{L^{\infty}\left([\tau, 2 \tau] ; H^{\sigma}\right)} \leqslant\left\|u(\tau)-\pi_{N} u_{N}(\tau)\right\|_{H^{\sigma}}+C \tau\left\|w_{N}\right\|_{L^{\infty}\left([-\tau, \tau] ; H^{\sigma}\right)}+\Lambda(N, v),
$$

which implies that

$$
\left\|w_{N}\right\|_{L^{\infty}\left([\tau, 2 \tau] ; H^{\sigma}\right)} \leqslant \Lambda(N, v) .
$$

Now we can cover the interval $[-t, t]$ by intervals of size $\tau$, and repeat the previous analysis to arrive at the bound

$$
\left\|u(t)-\pi_{N} u_{N}(t)\right\|_{H^{\sigma}} \leqslant \Lambda(N, v) .
$$


Next, we write

$$
u-u_{N}=u-\pi_{N} u_{N}-\left(1-\pi_{N}\right) u_{N} .
$$

We now invoke the following lemma.

Lemma 2.9. Fix $t \in \mathbb{R}$. For every $\varepsilon>0$ there is $N_{0}$ such that, for every $N \geqslant N_{0}$ and every $v \in K$,

$$
\left\|\left(1-\pi_{N}\right)\left(\Phi_{N}(t)(v)\right)\right\|_{H^{\sigma}}<\varepsilon .
$$

The proof of Lemma 2.9 is similar to (and simpler than) the proof of Lemma 2.8, and therefore will be omitted. We now come back to (2.9), and we use (2.8) and Lemma 2.9. This completes the proof of Proposition 2.7.

As a consequence of Proposition 2.7, we also have the following approximation property.

Proposition 2.10. Let $\sigma \geqslant \gamma / 2$. Fix $t \in \mathbb{R}, R>0$, and a compact $A \subset B_{R, \sigma}$. For every $\varepsilon>0$ there exists $N_{0}$ such that, for every $N \geqslant N_{0}$,

$$
\Phi(t)(A) \subset \Phi_{N}(t)\left(A+B_{\varepsilon, \sigma}\right) .
$$

Proof. Let $u \in \Phi(t)(A)$. This means that there exists $v \in A$ such that $u=$ $\Phi(t)(v)$. Write

$$
u=\Phi_{N}(t)\left(\Phi_{N}(-t) \Phi(t)(v)\right) .
$$

Set $w_{N}=\Phi_{N}(-t) \Phi(t)(v)$. The goal is to show that $w_{N} \in A+B_{\varepsilon, \sigma}$ for every $N \geqslant N_{0}(\varepsilon, t, A)$. For that purpose, we can write

$$
w_{N}=v+z_{N}, \quad z_{N} \equiv \Phi_{N}(-t) \Phi(t)(v)-v .
$$

Since $v \in A$, the issue is to check that $z_{N} \in B_{\varepsilon, \sigma}$ for every $N \geqslant N_{0}(\varepsilon, t, A)$. We can write

$$
z_{N}=\Phi_{N}(-t)\left(\Phi(t)(v)-\Phi_{N}(t)(v)\right) .
$$

Using Proposition 2.5, we obtain that

$$
\left\|\Phi_{N}(-t)\left(\Phi(t)(v)-\Phi_{N}(t)(v)\right)\right\|_{H^{\sigma}} \leqslant C(t, R)\left\|\Phi(t)(v)-\Phi_{N}(t)(v)\right\|_{H^{\sigma}} .
$$

Using Proposition 2.7, we obtain that

$$
\left\|\Phi(t)(v)-\Phi_{N}(t)(v)\right\|_{H^{\sigma}} \leqslant \Lambda(N, v),
$$

where for every $\varepsilon>0$ there is $N_{0}$ such that, for every $N \geqslant N_{0}$ and every $v \in A$, $\Lambda(N, v)<\varepsilon$. A combination of (2.10) and (2.11) implies that $\left\|z_{N}\right\|_{H^{\sigma}}<\varepsilon$, provided that $N \geqslant N_{0}(\varepsilon, t, A)$. This completes the proof of Proposition 2.10. 


\section{The case when $\gamma>2$ as a consequence of Ramer's result}

We will show in this section that in the case when $\gamma>2$ the result of Theorem 1.2 follows from [32]. Thanks to the Duhamel formula, we can write

$$
\Phi(t)=S(t) \circ \Psi(t)
$$

where

$$
\Psi(t)\left(u_{0}\right)=u_{0}-\int_{0}^{t} S(-\tau)\left(\left(1+\left|D_{x}\right|^{\gamma}\right)^{-1} \partial_{x}\left(\left(\Phi(\tau)\left(u_{0}\right)\right)^{2}\right)\right) d \tau .
$$

Thanks to the invariance of the complex Gaussians under rotations, the measure $\mu_{s}$ is invariant under $S(t)$ (see Lemma 4.3 below). Therefore, we need to show the quasiinvariance of $\mu_{s}$ under $\Psi(t)$. Take $\sigma_{1}>\frac{1}{2}$ to be chosen later. Write $\Psi(t)=$ Id $+K(t)$, where $K(t)$ is defined via (3.1). Thanks to [32] and the analysis of the previous section, the measure $\mu_{s}$ is quasiinvariant under $\Psi(t)$, if we can show that for $u_{0}$ in a bounded set of $H^{s+\gamma / 2-\sigma_{1}}$ and $|t| \leqslant 1$ small enough (depending only on the fixed bounded set) we have that the map $(D K(t))_{u_{0}}$ is a Hilbert-Schmidt map on $H^{s+\gamma / 2}$. After a direct computation we arrive at

$$
(D K(t))_{u_{0}}\left(v_{0}\right)=-2 \int_{0}^{t} S(-\tau)\left(\left(1+\left|D_{x}\right|^{\gamma}\right)^{-1} \partial_{x}\left(\Phi(\tau)\left(u_{0}\right) v(\tau)\right) d \tau,\right.
$$

where $v$ is a solution of the linear problem

$$
\partial_{t} v+\partial_{t}\left|D_{x}\right|^{\gamma} v+\partial_{x} v+2 \partial_{x}\left(\Phi(t)\left(u_{0}\right) v\right)=0,\left.\quad v\right|_{t=0}=v_{0} .
$$

Next, for $\sigma_{2}>1 / 2$ to be chosen later, we write

$$
(D K(t))_{u_{0}}=\left(1+\left|D_{x}\right|\right)^{-\sigma_{2}} \circ A,
$$

where

$$
A\left(v_{0}\right) \equiv-2 \int_{0}^{t} S(-\tau)\left(\left(1+\left|D_{x}\right|\right)^{\sigma_{2}}\left(1+\left|D_{x}\right|^{\gamma}\right)^{-1} \partial_{x}\left(\Phi(\tau)\left(u_{0}\right) v(\tau)\right) d \tau .\right.
$$

Since $\left(1+\left|D_{x}\right|\right)^{-\sigma_{2}}$ is a Hilbert-Schmidt map on $H^{s+\gamma / 2}$ and the Hilbert-Schmidt property is preserved by compositions with bounded maps, we are reduced to showing that the map $A$ is bounded on $H^{s+\gamma / 2}$. The assumption that $\gamma>2$ will be used in the verification of this property. Using (2.2), we can write

$$
\begin{aligned}
\left\|A\left(v_{0}\right)\right\|_{H^{s+\gamma / 2}} & \leqslant C \sup _{\tau \in[0, t]}\left\|\Phi(\tau)\left(u_{0}\right) v(\tau)\right\|_{H^{s-\gamma / 2+\sigma_{2}+1}} \\
& \leqslant C \sup _{\tau \in[0, t]}\left(\left\|\Phi(\tau)\left(u_{0}\right)\right\|_{H^{s-\gamma / 2+\sigma_{2}+1}}\|v(\tau)\|_{H^{s-\gamma / 2+\sigma_{2}+1}}\right) .
\end{aligned}
$$


We now estimate each of the factors. Thanks to the assumption that $\gamma>2$ for $\sigma_{1}$ and $\sigma_{2}$ close enough to $\frac{1}{2}$, we have

$$
s-\frac{\gamma}{2}+\sigma_{2}+1<s+\frac{\gamma}{2}-\sigma_{1}
$$

Therefore, using the results of the previous section, we obtain that, for $\tau \in[0, t]$,

$$
\left\|\Phi(\tau)\left(u_{0}\right)\right\|_{H^{s-\gamma / 2+\sigma_{2}+1}} \leqslant\left\|\Phi(\tau)\left(u_{0}\right)\right\|_{H^{s+\gamma / 2-\sigma_{1}}} \leqslant C\left\|u_{0}\right\|_{H^{s+\gamma / 2-\sigma_{1}}} .
$$

Next, coming back to (3.2), and using (2.2), we get that, for $\tau \in[0, t]$,

$$
\|v(\tau)\|_{H^{s-\gamma / 2+\sigma_{2}+1}} \leqslant\left\|v_{0}\right\|_{H^{s-\gamma / 2+\sigma_{2}+1}}+C|t| \sup _{\tau \in[0, t]}\left\|\Phi(\tau)\left(u_{0}\right) v(\tau)\right\|_{H^{s-\gamma / 2+\sigma_{2}+1}}
$$

(here we only use that $\gamma \geqslant 1$ ). Therefore using that, for $\sigma_{2}$ close enough to $\frac{1}{2}$, $s-\gamma / 2+\sigma_{2}+1<s+\gamma / 2$, we obtain

$$
\|v(\tau)\|_{H^{s-\gamma / 2+\sigma_{2}+1}} \leqslant\left\|v_{0}\right\|_{H^{s+\gamma / 2}}+C|t|\left\|u_{0}\right\|_{H^{s+\gamma / 2-\sigma_{1}}} \sup _{\tau \in[0, t]}\|v(\tau)\|_{H^{s-\gamma / 2+\sigma_{2}+1}} .
$$

Hence we get that, for $t$ small enough, but still only depending on the bounded set of $H^{s+\gamma / 2-\sigma_{1}}$ where $u_{0}$ ranges, one has the bound

$$
\|v(\tau)\|_{H^{s-\gamma / 2+\sigma_{2}+1}} \leqslant 2\left\|v_{0}\right\|_{H^{s+\gamma / 2}}, \quad \tau \in[0, t] .
$$

Therefore, we obtain that the map $A$ is bounded on $H^{s+\gamma / 2}$. This in turn implies that $(D K(t))_{u_{0}}$ is a Hilbert-Schmidt map, and consequently we can apply the result of [32] to get the result of Theorem 1.2 for $\gamma>2$.

From now on we shall suppose that $\gamma \in\left(\frac{4}{3}, 2\right]$. This is the range of $\gamma$ which does not seem covered by [32]. In this region of $\gamma$, we shall use more involved properties of the transformation $\Phi(t)$. These considerations seem to go beyond the analysis of general maps close to the identity done in [32]. Let us also mention that the result of [32] under the Hilbert-Schmidt assumption is already a quite nontrivial result using a stochastic interpretation of the obtained densities (the straightforward result being obtained under a trace class assumption).

\section{A variable change formula}

For every $N$, we denote by $E_{N}$ the real vector space spanned by

$$
(\cos (n x), \sin (n x))_{1 \leqslant n \leqslant N} .
$$

We equip $E_{N}$ with the natural scalar product. We endow $E_{N}$ with a Lebesgue measure $L_{N}$ as follows. If

$$
\left(\pi_{N} u\right)(x)=\sum_{0<|n| \leqslant N} u_{n} e^{i n x}, \quad u_{n}=\overline{u_{-n}}
$$


and $u_{n}=a_{n}+i b_{n},\left(a_{n}, b_{n}\right) \in \mathbb{R}^{2}$, then

$$
\left(\pi_{N} u\right)(x)=\sum_{n=1}^{N}\left(a_{n}(2 \cos (n x))+b_{n}(-2 \sin (n x))\right) .
$$

Therefore, we denote by $L_{N}$ the Lebesgue measure on $E_{N}$ built with respect to the orthogonal basis

$$
(2 \cos (n x),-2 \sin (n x))_{1 \leqslant n \leqslant N} .
$$

Next, we denote by $E_{N}^{\perp}$ the orthogonal complement of $E_{N}$ in $H^{s}$. We endow $E_{N}^{\perp}$ with the measure $\mu_{s ; N}^{\perp}$, which is the image measure under the map

$$
\omega \longmapsto \sum_{|n|>N} \frac{g_{n}(\omega)}{|n|^{s+\gamma / 2}} e^{i n x} .
$$

We can now see the measure $\mu_{s}$ as a product measure on $E_{N} \times E_{N}^{\perp}$ as follows:

$$
\begin{aligned}
d \mu_{s} & =\gamma_{N} e^{-\sum_{n=1}^{N}|n|^{2 s+\gamma}\left(a_{n}^{2}+b_{n}^{2}\right)} d L_{N}\left(a_{1}, b_{1}, \ldots, a_{N}, b_{N}\right) d \mu_{s ; N}^{\perp} \\
& =\gamma_{N} e^{-\left\|\pi_{N} u\right\|_{H^{s+\gamma / 2}}^{2}} d u_{1} \ldots d u_{N} d \mu_{s ; N}^{\perp},
\end{aligned}
$$

where $\gamma_{N}$ is a suitable renormalization factor and

$$
\left(\pi_{N} u\right)(x)=\sum_{0<|n| \leqslant N} u_{n} e^{i n x}, \quad u_{n}=\overline{u_{-n}}, \quad u_{n}=a_{n}+i b_{n},\left(a_{n}, b_{n}\right) \in \mathbb{R}^{2} .
$$

We have the following 'change of variables rule'.

Proposition 4.1. For A a Borel set of $H^{s}$, one has the identity

$$
\begin{aligned}
\mu_{s, r}\left(\Phi_{N}(t)(A)\right) & =\int_{\Phi_{N}(t)(A)} \chi_{r}(u) d \mu_{s}(u) \\
& =\gamma_{N} \int_{A} \chi_{r}(u) e^{-\left\|\pi_{N}\left(\Phi_{N}(t)(u)\right)\right\|_{H^{s+\gamma / 2}}^{2}} d u_{1} \ldots d u_{N} d \mu_{s ; N}^{\perp} .
\end{aligned}
$$

Proof. We follow [36]. A difference with [36] is that, in Proposition 4.1, we deal with $\chi_{r}(u)$ and not $\chi_{r}\left(\pi_{N} u\right)$. As we will see below, thanks to the conservation law of Lemma 2.4, the analysis is not affected by the lack of the projector $\pi_{N}$. Let us denote by $\tilde{\Phi}_{N}(t)$ the (well-defined) flow of the following ordinary differential equation (ODE) on $E_{N}$,

$$
\partial_{t} u+\partial_{t}\left|D_{x}\right|^{\gamma} u+\partial_{x} u+\partial_{x} \pi_{N}\left(u^{2}\right)=0, \quad u(0, x) \in E_{N} .
$$


Observe that, since for the solution of (4.1) we have $u=\pi_{N} u$, we also have that $\partial_{x} \pi_{N}\left(u^{2}\right)=\partial_{x} \pi_{N}\left(\left(\pi_{N} u\right)^{2}\right)$. Therefore, we have the following relation:

$$
\Phi_{N}(t)\left(u_{0}\right)=\tilde{\Phi}_{N}(t)\left(\pi_{N} u_{0}\right)+S(t)\left(\left(1-\pi_{N}\right) u_{0}\right) .
$$

We have the following lemma.

LEMMA 4.2. The measure $d u_{1} \ldots d u_{N}$ is invariant under the flow $\tilde{\Phi}_{N}(t)$.

Proof. If

$$
u(x)=\sum_{0<|n| \leqslant N} u_{n} e^{i n x}, \quad u_{n}=\overline{u_{-n}},
$$

then Equation (4.1) can be rewritten as

$$
\partial_{t} u_{n}=-\frac{i n}{1+|n|^{\gamma}}\left(u_{n}+\sum_{\substack{n_{1}+n_{2}=n \\ 0<\left|n_{1}\right|,\left|n_{2}\right| \leqslant N}} u_{n_{1}} u_{n_{2}}\right), \quad 1 \leqslant n \leqslant N,
$$

and thus, if $u_{n}=a_{n}+i b_{n}$, we arrive at the equations

$$
\begin{aligned}
\partial_{t} a_{n} & =\frac{n}{1+|n|^{\gamma}}\left(b_{n}+\sum_{\substack{n_{1}+n_{2}=n \\
0<\left|n_{1}\right|,\left|n_{2}\right| \leqslant N}}\left(a_{n_{1}} b_{n_{2}}+a_{n_{2}} b_{n_{1}}\right)\right), \\
\partial_{t} b_{n} & =-\frac{n}{1+|n|^{\gamma}}\left(a_{n}+\sum_{\substack{n_{1}+n_{2}=n \\
0<\left|n_{1}\right|,\left|n_{2}\right| \leqslant N}}\left(a_{n_{1}} a_{n_{2}}-b_{n_{1}} b_{n_{2}}\right)\right) .
\end{aligned}
$$

We now observe that if we write the last equations as

$$
\partial_{t} a_{n}=F_{n}\left(a_{1}, \ldots, a_{N}, b_{1} \ldots, b_{N}\right), \quad \partial_{t} b_{n}=G_{n}\left(a_{1}, \ldots, a_{N}, b_{1} \ldots, b_{N}\right),
$$

then we have the remarkable property that

$$
\frac{\partial F_{n}}{\partial a_{n}}=\frac{\partial G_{n}}{\partial b_{n}}=0, \quad 1 \leqslant n \leqslant N .
$$

In particular, the ODE (4.1) is generated by a divergence-free vector field. Therefore the statement of Lemma 4.2 follows from the Liouville theorem.

We also have the following statement.

LEMMA 4.3. The measure $\mu_{s ; N}^{\perp}$ on $E_{N}^{\perp}$ is invariant under the map $S(t)$. In particular, $\mu_{s}$ is invariant under $S(t)$. 
Proof. The proof of Lemma 4.3 follows from the invariance of the complex Gaussian under rotations. We follow closely [36, Lemma 5.3], where the proof of an analogous statement is given. For $M>N$, we denote by $E_{N}^{M}$ the finitedimensional real vector space spanned by $(\cos (n x), \sin (n x))$, where $N<n \leqslant M$. We denote by $\mu_{N}^{M}$ the centred Gaussian measure on $E_{N}^{M}$ induced by the series

$$
\sum_{|n|=N+1}^{M} \frac{g_{n}(\omega)}{|n|^{s+\gamma / 2}} e^{i n x} .
$$

By using the Fatou lemma, we obtain that, if $U$ is an open set of $E_{N}^{\perp}$, then we have

$$
\mu_{N}^{\perp}(U) \leqslant \liminf _{M \rightarrow \infty} \mu_{N}^{M}\left(U \cap E_{N}^{M}\right) .
$$

By passing to a complementary set in (4.3), we get that, for $F$ a closed set of $E_{N}^{\perp}$,

$$
\mu_{N}^{\perp}(F) \geqslant \limsup _{M \rightarrow \infty} \mu_{N}^{M}\left(F \cap E_{N}^{M}\right) .
$$

By the definition of $S(t)$, we get

$$
\begin{aligned}
& S(t)(\cos (n x))=\cos \left(-\frac{t n}{1+|n|^{\gamma}}+n x\right), \\
& S(t)(\sin (n x))=\sin \left(-\frac{t n}{1+|n|^{\gamma}}+n x\right) .
\end{aligned}
$$

Therefore, for fixed $t$, the map $S(t)$ acts as a rotation on the vector space spanned by $\cos (n x)$ and $\sin (n x)$. Consequently, by the invariance of centred Gaussians by rotations, we obtain that the measure $\mu_{N}^{M}$ is invariant under $S(t)$. Let $F$ be a closed set of $E_{N}^{\perp}$. Then $S(t)(F)$ is also closed, and, thanks to (4.4),

$$
\mu_{N}^{\perp}\left(S(t)(F)+\overline{B_{\varepsilon}}\right) \geqslant \limsup _{M \rightarrow \infty} \mu_{N}^{M}\left(\left(S(t) F+\overline{B_{\varepsilon}}\right) \cap E_{N}^{M}\right),
$$

where $B_{\varepsilon}$ denotes the open ball of radius $\varepsilon$ in $E_{N}^{\perp}\left(E_{N}^{\perp}\right.$ is equipped with the $H^{s}$ topology). Using that $S(t)$ acts as an isometry on $H^{s}$ and the invariance of $E_{N}^{M}$ under $S(t)$, we obtain that, for every $\varepsilon$ and every $M$,

$$
S(t)\left(\left(F+B_{\varepsilon}\right) \cap E_{N}^{M}\right) \subset\left(S(t) F+\overline{B_{\varepsilon}}\right) \cap E_{N}^{M} .
$$

Therefore, using the invariance of $\mu_{N}^{M}$ under $S(t)$ and (4.3), we get

$$
\begin{aligned}
\mu_{N}^{\perp}\left(S(t)(F)+\overline{B_{\varepsilon}}\right) & \geqslant \limsup _{M \rightarrow \infty} \mu_{N}^{M}\left(S(t)\left(\left(F+B_{\varepsilon}\right) \cap E_{N}^{M}\right)\right) \\
& =\limsup _{M \rightarrow \infty} \mu_{N}^{M}\left(\left(F+B_{\varepsilon}\right) \cap E_{N}^{M}\right) \\
& \geqslant \mu_{N}^{\perp}\left(F+B_{\varepsilon}\right) \geqslant \mu_{N}^{\perp}(F) .
\end{aligned}
$$


Letting $\varepsilon \rightarrow 0$, and using the Lebesgue theorem, we get $\mu_{N}^{\perp}(F) \leqslant \mu_{N}^{\perp}(S(t)(F))$. By the time reversibility of $S(t)$, we get $\mu_{N}^{\perp}(F)=\mu_{N}^{\perp}(S(t)(F))$ for every closed set $F$ of $E^{N}$. Finally, by approximation arguments, we obtain that $\mu_{N}^{\perp}(A)=\mu_{N}^{\perp}(S(t)(A))$ for every Borel set $A$ of $E_{N}^{\perp}$. This completes the proof of Lemma 4.3.

Let us now complete the proof of Proposition 4.1. Again, we follow closely [36]. Recall that $d L_{N}=d u_{1} \ldots d u_{N}$. We can write

$$
\int_{\Phi_{N}(t)(A)} \chi_{r}(u) e^{-\left\|\pi_{N} u\right\|_{H^{s+\gamma / 2}}^{2}} d L_{N} d \mu_{s ; N}^{\perp}
$$

as

$$
\int_{E_{N}} \int_{E_{N}^{\perp}} 1\left(\Phi_{N}(t)(A)\right)(u) \chi_{r}(u) e^{-\left\|\pi_{N} u\right\|_{H^{s+\gamma / 2}}^{2}} d L_{N} d \mu_{s ; N}^{\perp},
$$

where 1 denotes the indicator function of a measurable set. Using the Fubini theorem, we obtain that (4.5) can be written as

$$
\begin{aligned}
& \int_{E_{N}} e^{-\left\|\pi_{N} u\right\|_{H^{s+\gamma / 2}}^{2}}\left(\int _ { E _ { N } ^ { \perp } } 1 ( \Phi _ { N } ( t ) ( A ) ) \left(\pi_{N}(u)\right.\right. \\
& \left.\left.+\pi_{>N}(u)\right) \chi_{r}\left(\pi_{N}(u)+\pi_{>N}(u)\right) d \mu_{s ; N}^{\perp}\right) d L_{N},
\end{aligned}
$$

where $\pi_{>N}=\mathrm{Id}-\pi_{N}$. Thanks to Lemma 4.3, we can write the last expression as

$$
\begin{aligned}
& \int_{E_{N}} e^{-\left\|\pi_{N} u\right\|_{H^{s+\gamma / 2}}^{2}}\left(\int _ { E _ { N } ^ { \perp } } 1 ( \Phi _ { N } ( t ) ( A ) ) \left(\pi_{N}(u)\right.\right. \\
& \left.\left.\quad+S(t) \pi_{>N}(u)\right) \chi_{r}\left(\pi_{N}(u)+S(t) \pi_{>N}(u)\right) d \mu_{s ; N}^{\perp}\right) d L_{N} .
\end{aligned}
$$

Using once again the Fubini theorem, we get that (4.5) is equal to

$$
\begin{aligned}
& \int_{E_{N}^{\perp}}\left(\int _ { E _ { N } } e ^ { - \| \pi _ { N } u \| _ { H ^ { s + \gamma / 2 } } ^ { 2 } } 1 ( \Phi _ { N } ( t ) ( A ) ) \left(\pi_{N}(u)\right.\right. \\
& \left.\left.\quad+S(t) \pi_{>N}(u)\right) \chi_{r}\left(\pi_{N}(u)+S(t) \pi_{>N}(u)\right) d L_{N}\right) d \mu_{s ; N}^{\perp} .
\end{aligned}
$$

Now, using Lemma 4.2, we obtain that the last expression is equal to

$$
\begin{aligned}
\int_{E_{N}^{\perp}}\left(\int _ { E _ { N } } e ^ { - \| \tilde { \Phi } _ { N } ( t ) ( \pi _ { N } u ) \| _ { H ^ { s + \gamma / 2 } } ^ { 2 } } 1 ( \Phi _ { N } ( t ) ( A ) ) \left(\tilde{\Phi}_{N}(t)\left(\pi_{N} u\right)\right.\right. \\
\left.\left.\quad+S(t) \pi_{>N}(u)\right) \chi_{r}\left(\tilde{\Phi}_{N}(t)\left(\pi_{N} u\right)+S(t) \pi_{>N}(u)\right) d L_{N}\right) d \mu_{s ; N}^{\perp} .
\end{aligned}
$$


Coming back to (4.2), we observe that $\tilde{\Phi}_{N}(t)\left(\pi_{N} u\right)=\pi_{N} \Phi_{N}(t)(u)$, and therefore (4.5) is equal to

$$
\int_{E_{N}^{\perp}}\left(\int_{E_{N}} e^{-\left\|\pi_{N} \Phi_{N}(t)(u)\right\|_{H^{s+\gamma / 2}}^{2}} 1\left(\Phi_{N}(t)(A)\right)\left(\Phi_{N}(t)(u)\right) \chi_{r}\left(\Phi_{N}(t)(u)\right) d L_{N}\right) d \mu_{s ; N}^{\perp} .
$$

Since $\Phi_{N}(t)$ is a bijection, we have that $1\left(\Phi_{N}(t)(A)\right)\left(\Phi_{N}(t)(u)\right)=1(A)(u)$. Moreover, using Lemma 2.4, we obtain that $\chi_{r}\left(\Phi_{N}(t)(u)\right)=\chi_{r}(u)$. Therefore, we finally obtain that (4.5) is equal to

$$
\int_{E_{N}^{\perp}}\left(\int_{E_{N}} e^{-\left\|\pi_{N} \Phi_{N}(t)(u)\right\|_{H^{s+\gamma / 2}}^{2}} 1(A)(u) \chi_{r}(u) d L_{N}\right) d \mu_{s ; N}^{\perp},
$$

which is equal to

$$
\int_{A} \chi_{r}(u) e^{-\left\|\pi_{N}\left(\Phi_{N}(t)(u)\right)\right\|_{H^{s+\gamma / 2}}^{2}} d u_{1} \ldots d u_{N} d \mu_{s ; N}^{\perp} .
$$

This completes the proof of Proposition 4.1.

\section{An energy estimate}

The following energy estimate is of importance in the study of the transport of the measure $\mu_{s}$ by $\Phi(t)$.

Proposition 5.1. Let $\gamma \in\left(\frac{4}{3}, 2\right]$, and let $s \geqslant 1$. Then there exist $\kappa<2, \varepsilon>0$, and a constant $C$ such that, for every $N$ and every solution $u$ of (2.1),

$$
\frac{d}{d t}\left\|\pi_{N} u(t)\right\|_{H^{s+\gamma / 2}}^{2} \leqslant C\left(1+\left\|\pi_{N} u(t)\right\|_{H^{\gamma / 2}}^{3-\kappa}\right)\left(1+\left\|\left|D_{x}\right|^{s+\gamma / 2-1 / 2-\varepsilon} \pi_{N} u(t)\right\|_{L^{\infty}}^{\kappa}\right) .
$$

Proof. We first observe that $\pi_{N} u$ is a solution of

$$
\partial_{t} \pi_{N} u+\partial_{t}\left|D_{x}\right|^{\gamma} \pi_{N} u+\partial_{x} \pi_{N} u+\partial_{x} \pi_{N}\left(\left(\pi_{N} u\right)^{2}\right)=0 .
$$

Therefore, we can compute

$$
\begin{aligned}
\frac{d}{d t}\left\|\pi_{N} u(t)\right\|_{H^{s+\gamma / 2}}^{2}= & \frac{1}{2 \pi} \int\left(\left|D_{x}\right|^{s+\gamma / 2} \pi_{N} u\right)\left|D_{x}\right|^{s+\gamma / 2}\left(-\left(1+\left|D_{x}\right|^{\gamma}\right)^{-1} \partial_{x} \pi_{N} u\right. \\
& \left.-\left(1+\left|D_{x}\right|^{\gamma}\right)^{-1} \partial_{x} \pi_{N}\left(\pi_{N} u\right)^{2}\right) .
\end{aligned}
$$

Since

$$
\int\left(\left|D_{x}\right|^{s+\gamma / 2} \pi_{N} u\right)\left(\left|D_{x}\right|^{s+\gamma / 2}\left(1+\left|D_{x}\right|^{\gamma}\right)^{-1} \partial_{x} \pi_{N} u\right)=0
$$


and using that $\pi_{N}$ is a projector, we obtain that

$$
\begin{aligned}
\frac{d}{d t}\left\|\pi_{N} u(t)\right\|_{H^{s+\gamma / 2}}^{2} & =-\frac{1}{2 \pi} \int\left(\left|D_{x}\right|^{s+\gamma / 2} \pi_{N} u\right)\left|D_{x}\right|^{s+\gamma / 2}\left(\left(1+\left|D_{x}\right|^{\gamma}\right)^{-1} \partial_{x}\left(\pi_{N} u\right)^{2}\right) \\
& =-\frac{1}{2 \pi} \int\left(\left(1+\left|D_{x}\right|^{\gamma}\right)^{-1}\left|D_{x}\right|^{s+\gamma} \pi_{N} u\right)\left|D_{x}\right|^{s}\left(\partial_{x}\left(\pi_{N} u\right)^{2}\right) .
\end{aligned}
$$

By writing

$$
\left(1+\left|D_{x}\right|^{\gamma}\right)^{-1}\left|D_{x}\right|^{\gamma}=\mathrm{Id}-\left(1+\left|D_{x}\right|^{\gamma}\right)^{-1}
$$

we arrive at

$$
\frac{d}{d t}\left\|\pi_{N} u(t)\right\|_{H^{s+\gamma / 2}}^{2}=I_{1}+I_{2}
$$

where

$$
I_{1}=-\frac{1}{2 \pi} \int\left(\left|D_{x}\right|^{s} \pi_{N} u\right)\left|D_{x}\right|^{s}\left(\partial_{x}\left(\pi_{N} u\right)^{2}\right)=-\frac{1}{2 \pi} \int\left(\partial_{x}^{s} \pi_{N} u\right) \partial_{x}^{s}\left(\partial_{x}\left(\pi_{N} u\right)^{2}\right)
$$

and

$$
I_{2}=\frac{1}{2 \pi} \int\left(\left(1+\left|D_{x}\right|^{\gamma}\right)^{-1}\left|D_{x}\right|^{s} \pi_{N} u\right)\left|D_{x}\right|^{s}\left(\partial_{x}\left(\pi_{N} u\right)^{2}\right) .
$$

Let us first estimate the more regular contribution of $I_{2}$. Using that $\gamma>1$ and $s>1 / 2$, we obtain that

$$
I_{2} \lesssim\left\|\pi_{N} u\right\|_{H^{s}}\left\|\left(\pi_{N} u\right)^{2}\right\|_{H^{s}} \lesssim\left\|\pi_{N} u\right\|_{H^{s}}^{2}\left\|\pi_{N} u\right\|_{L^{\infty}} \lesssim\left\|\pi_{N} u\right\|_{H^{s}}^{2}\left\|\pi_{N} u\right\|_{H^{\gamma / 2}} .
$$

Thanks to a suitable use of the Hölder inequality, we obtain that, for some $\theta>0$,

$$
\left\|\pi_{N} u\right\|_{H^{s}} \leqslant\left\|\pi_{N} u\right\|_{H^{\gamma / 2}}^{\theta}\left\|\pi_{N} u\right\|_{H^{s+\gamma / 2-1 / 2-\varepsilon}}^{1-\theta},
$$

provided that $\varepsilon$ is small enough. Now, since our spatial domain is compact, we have that

$$
\left\|\pi_{N} u\right\|_{H^{s+\gamma / 2-1 / 2-\varepsilon}} \lesssim\left\|\left|D_{x}\right|^{s+\gamma / 2-1 / 2-\varepsilon} \pi_{N} u\right\|_{L^{\infty}} .
$$

Therefore we arrive at

$$
I_{2} \lesssim\left\|\pi_{N} u\right\|_{H^{\gamma / 2}}^{1+2 \theta}\left\|\left|D_{x}\right|^{s+\gamma / 2-1 / 2-\varepsilon} \pi_{N} u\right\|_{L^{\infty}}^{2-2 \theta},
$$

which is an acceptable bound.

Let us next turn to the more delicate analysis of $I_{1}$. We can write

$$
I_{1}=-\frac{1}{\pi} \int \partial_{x}^{s} v \partial_{x}^{s}\left(\partial_{x} v v\right)
$$


where, for brevity, we set $v=\pi_{N} u$. When applying the Leibniz rule, the most delicate term which appears is the one when all $s$ derivatives hit on $\partial_{x} v$. Namely, we have to deal with the term

$$
\int\left(\partial_{x}^{s} v\right)\left(\partial_{x}^{s+1} v\right) v
$$

In the spirit of the local well-posedness theory of quasilinear hyperbolic PDEs, the main observation is that one may rewrite (5.1) as

$$
-\frac{1}{2} \int \partial_{x} v\left(\partial_{x}^{s} v\right)^{2}
$$

Therefore, thanks to the last key argument and the Leibniz rule, we obtain that, in order to estimate $I_{1}$, it suffices to estimate the expressions

$$
\int\left(\partial_{x}^{s} v\right)\left(\partial_{x}^{\sigma_{1}} v\right)\left(\partial_{x}^{\sigma_{2}} v\right)
$$

where

$$
\sigma_{1}+\sigma_{2}=s+1, \quad \sigma_{1} \leqslant s, \sigma_{2} \leqslant s
$$

(the important point being that $\sigma_{1}$ and $\sigma_{2}$ are not allowed to be $s+1$ ). For that purpose, we will use the following lemma.

Lemma 5.2. Let $\sigma \in[\gamma / 2, s+\gamma / 2-1 / 2-\varepsilon]$. Suppose that $\theta \in[0,1]$ is such that

$$
\sigma<\theta \frac{\gamma}{2}+(1-\theta)\left(s+\frac{\gamma}{2}-\frac{1}{2}-\varepsilon\right) \text {. }
$$

Then, for $u$ such that $\hat{u}(0)=0$, we have the bound

$$
\left\|\partial_{x}^{\sigma} u\right\|_{L^{p}} \lesssim\|u\|_{H^{\gamma / 2}}^{\theta}\left\|\left|D_{x}\right|^{s+\gamma / 2-1 / 2-\varepsilon} u\right\|_{L^{\infty}}^{1-\theta},
$$

provided that $\varepsilon>0$ is sufficiently small, where $1 / p=\theta / 2+(1-\theta) / \infty$; that is, $p=2 / \theta$.

Proof. Consider a Littlewood-Paley decomposition of the unity

$$
\mathrm{Id}=\sum_{\lambda} \Delta_{\lambda}
$$

where the summation is taken over the dyadic values of $\lambda$, that is, $\lambda=2^{j}, j=0,1$, $2, \ldots$, and $\Delta_{\lambda}$ are Littlewood-Paley projectors. More precisely, they are defined as Fourier multipliers as $\Delta_{0}=\psi_{0}\left(\left|D_{x}\right|\right)$, and, for $\lambda \geqslant 1, \Delta_{\lambda}=\psi\left(\left|D_{x}\right| / \lambda\right)$, 
where $\psi_{0} \in C_{0}^{\infty}\left(-\frac{1}{2}, \frac{1}{2}\right)$ and $\psi \in C_{0}^{\infty}(\mathbb{R} \backslash\{0\})$ are suitable functions such that (5.4) holds. In what follows, we shall use that for every $\varphi \in C_{0}^{\infty}(\mathbb{R} \backslash\{0\})$ one has the bound

$$
\left\|\varphi\left(\left|D_{x}\right| / \lambda\right)(f)\right\|_{L^{p}} \leqslant C\|f\|_{L^{p}}, \quad p \in[1, \infty], \lambda \geqslant 1,
$$

where the constant $C$ is independent of $\lambda$. The bound (5.5) is a consequence of the Schur lemma. Indeed, one needs to invoke the following estimate for the kernel of $\varphi\left(\left|D_{x}\right| / \lambda\right)$,

$$
\left|\sum_{n} \varphi\left(\frac{|n|}{\lambda}\right) e^{i n(x-y)}\right| \leqslant \frac{C \lambda}{(1+\lambda|x-y|)^{2}},
$$

which follows after two summations by parts. We notice that the extension of (5.5) when the circle is replaced by a compact Riemannian manifold is known to hold (see, for example, [12]).

For $u$ such that $\hat{u}(0)=0$, we have $\Delta_{0}(u)=0$, and therefore

$$
\left\|\partial_{x}^{\sigma} u\right\|_{L^{p}} \leqslant \sum_{\lambda \geqslant 1}\left\|\partial_{x}^{\sigma} \Delta_{\lambda} u\right\|_{L^{p}}
$$

Similarly to (5.5), using the Schur lemma, we can write

$$
\left\|\partial_{x}^{\sigma} \Delta_{\lambda} u\right\|_{L^{p}} \lesssim \lambda^{\sigma}\left\|\Delta_{\lambda} u\right\|_{L^{p}} .
$$

Using (5.6), (5.7), and the Hölder inequality, we arrive at the bound

$$
\left\|\partial_{x}^{\sigma} u\right\|_{L^{p}} \lesssim \sum_{\lambda \geqslant 1} \lambda^{\sigma}\left\|\Delta_{\lambda} u\right\|_{L^{2}}^{\theta}\left\|\Delta_{\lambda} u\right\|_{L^{\infty}}^{1-\theta}
$$

where $p=2 / \theta$. Now we can write

$$
\lambda^{s+\gamma / 2-1 / 2-\varepsilon} \Delta_{\lambda}=\tilde{\psi}\left(\left|D_{x}\right| / \lambda\right)\left|D_{x}\right|^{s+\gamma / 2-1 / 2-\varepsilon},
$$

where $\tilde{\psi} \in C_{0}^{\infty}(\mathbb{R} \backslash\{0\})$ is chosen such that, for $x \geqslant 0, \tilde{\psi}(x)=\psi(x) / x^{s+\gamma / 2-1 / 2-\varepsilon}$. Therefore, using (5.5), we get

$$
\left\|\Delta_{\lambda} u\right\|_{L^{\infty}} \lesssim \lambda^{-(s+\gamma / 2-1 / 2-\varepsilon)}\left\|\left|D_{x}\right|^{s+\gamma / 2-1 / 2-\varepsilon} u\right\|_{L^{\infty}} .
$$

Similarly, one obtains

$$
\left\|\Delta_{\lambda} u\right\|_{L^{2}} \lesssim \lambda^{-\gamma / 2}\left\|\left|D_{x}\right|^{\gamma / 2} u\right\|_{L^{2}} .
$$

Therefore, coming back to (5.8), we get the bound

$$
\left\|\partial_{x}^{\sigma} u\right\|_{L^{p}} \lesssim\left\|\left|D_{x}\right|^{\gamma / 2} u\right\|_{L^{2}}^{\theta}\left\|\left|D_{x}\right|^{s+\gamma / 2-1 / 2-\varepsilon} u\right\|_{L^{\infty}}^{1-\theta}\left(\sum_{\lambda \geqslant 1} \lambda^{\sigma-\theta(\gamma / 2)-(1-\theta)(s+\gamma / 2-1 / 2-\varepsilon)}\right) .
$$


Thanks to (5.3), the sum appearing in the right-hand side of the last inequality is convergent. Thus we arrive at the required bound. This completes the proof of Lemma 5.2.

Let us estimate the expressions (5.2). Suppose first that $s \geqslant 2$. For $\varepsilon_{1}$ and $\varepsilon$ sufficiently small to be fixed, we define the numbers $\theta_{1}, \theta_{2}, \theta_{3}$ in the interval $(0,1)$ as

$$
\begin{aligned}
s+\varepsilon_{1} & =\theta_{1} \frac{\gamma}{2}+\left(1-\theta_{1}\right)\left(s+\frac{\gamma}{2}-\frac{1}{2}-\varepsilon\right) \\
\sigma_{1}+\varepsilon_{1} & =\theta_{2} \frac{\gamma}{2}+\left(1-\theta_{2}\right)\left(s+\frac{\gamma}{2}-\frac{1}{2}-\varepsilon\right) \\
\sigma_{2}+\varepsilon_{1} & =\theta_{3} \frac{\gamma}{2}+\left(1-\theta_{3}\right)\left(s+\frac{\gamma}{2}-\frac{1}{2}-\varepsilon\right)
\end{aligned}
$$

(observe that $s, \sigma_{1}, \sigma_{2} \in[1, s]$ and $1 / 2 \leqslant \gamma / 2 \leqslant 1$ ). We next define $p_{1}, p_{2}, p_{3}$ as $p_{j}=2 / \theta_{j}, j=1,2,3$. We now check that, under our assumptions of $\gamma$ and $s$ for $\varepsilon_{1}$ and $\varepsilon$ sufficiently small, we have

$$
\frac{1}{p_{1}}+\frac{1}{p_{2}}+\frac{1}{p_{3}} \leqslant 1
$$

Clearly (5.12) is equivalent to $\theta_{1}+\theta_{2}+\theta_{3} \leqslant 2$. But coming back to (5.9)-(5.11), and using that $\sigma_{1}+\sigma_{2}=s+1$, we obtain that

$$
\theta_{1}+\theta_{2}+\theta_{3}=\frac{s+\frac{3 \gamma}{2}-\frac{5}{2}-3 \varepsilon_{1}-3 \varepsilon}{s-\frac{1}{2}-\varepsilon} .
$$

Therefore, for $\varepsilon_{1}$ and $\varepsilon$ sufficiently small, condition (5.12) follows from $s+3 / 2>$ $3 \gamma / 2$, which is satisfied thanks to the assumption that $s \geqslant 2$.

Thanks to (5.12), we can apply the Hölder inequality and Lemma 5.2 to write

$$
\begin{aligned}
\left|\int\left(\partial_{x}^{s} v\right)\left(\partial_{x}^{\sigma_{1}} v\right)\left(\partial_{x}^{\sigma_{2}} v\right)\right| & \leqslant\left\|\partial_{x}^{s} v\right\|_{L^{p_{1}}}\left\|\partial_{x}^{\sigma_{1}} v\right\|_{L^{p_{2}}}\left\|\partial_{x}^{\sigma_{2}} v\right\|_{L^{p_{3}}} \\
& \lesssim\|v\|_{H^{\gamma / 2}}^{\theta_{1}+\theta_{2}+\theta_{3}}\left\|\left|D_{x}\right|^{s+\gamma / 2-1 / 2-\varepsilon} v\right\|_{L^{\infty}}^{3-\theta_{1}-\theta_{2}-\theta_{3}}
\end{aligned}
$$

Therefore it remains to verify that $\theta_{1}+\theta_{2}+\theta_{3}>1$. But for $\varepsilon_{1}$ and $\varepsilon$ sufficiently small this condition follows from our assumption that $\gamma>\frac{4}{3}$.

Let us finally consider the case when $s=1$, which is not covered by the above analysis. In this case, we only need to estimate $\int\left(\partial_{x} v\right)^{3}$. Therefore, in the case when $s=1$ one gets an acceptable bound for $I_{1}$ thanks to the following lemma. 
LEMMA 5.3. For every $\gamma>\frac{4}{3}$ there is $\theta>\frac{1}{3}$ such that, for $u$ satisfying $\hat{u}(0)=0$, we have the bound

$$
\left\|\partial_{x} u\right\|_{L^{3}} \lesssim\|u\|_{H^{\gamma / 2}}^{\theta}\left\|\left|D_{x}\right|^{1 / 2+\gamma / 2-\varepsilon} u\right\|_{L^{\infty}}^{1-\theta},
$$

provided that $\varepsilon>0$ is sufficiently small.

Proof. As in the proof of Lemma 5.2, we perform a Littlewood-Paley decomposition. We can write

$$
\left\|\partial_{x} u\right\|_{L^{3}} \lesssim \sum_{\lambda \geqslant 1} \lambda\left\|\Delta_{\lambda} u\right\|_{L^{2}}^{2 / 3}\left\|\Delta_{\lambda} u\right\|_{L^{\infty}}^{1 / 3}
$$

We now choose $\sigma$ such that

$$
\frac{2}{3} \sigma+\frac{1}{3}\left(\frac{1}{2}+\frac{\gamma}{2}-\varepsilon\right)>1 \text {. }
$$

Thanks to (5.13) as in the proof of Lemma 5.2, we arrive at the bound

$$
\left\|\partial_{x} u\right\|_{L^{3}} \lesssim\|u\|_{H^{\sigma}}^{2 / 3}\left\|\left|D_{x}\right|^{1 / 2+\gamma / 2-\varepsilon} u\right\|_{L^{\infty}}^{1 / 3} .
$$

We choose $\sigma$ more precisely such that

$$
\frac{2}{3} \sigma+\frac{1}{3}\left(\frac{1}{2}+\frac{\gamma}{2}-\varepsilon\right)=1+\varepsilon,
$$

which leads to

$$
\sigma=\frac{5-\gamma+8 \varepsilon}{4}
$$

We first observe that for $\gamma>1$, we have $\sigma<1 / 2+\gamma / 2-\varepsilon$, provided $\varepsilon$ is small enough. If $\sigma \leqslant \gamma / 2$ (that is, $\gamma>\frac{5}{3}$ ) then the bound (5.14) is already sufficient to complete the proof of Lemma 5.3 (with $\theta=\frac{2}{3}$ ). We can therefore suppose that $\sigma \in[\gamma / 2,1 / 2+\gamma / 2-\varepsilon]$. Now, thanks to a suitable use of the Hölder inequality, we can write

$$
\|u\|_{H^{\sigma}} \leqslant\|u\|_{H^{\gamma / 2}}^{\alpha}\|u\|_{H^{1 / 2+\gamma / 2-\varepsilon}}^{1-\alpha} \lesssim\|u\|_{H^{\gamma / 2}}^{\alpha}\left\|\left|D_{x}\right|^{1 / 2+\gamma / 2-\varepsilon} u\right\|_{L^{\infty}}^{1-\alpha},
$$

where

$$
\sigma=\alpha \frac{\gamma}{2}+(1-\alpha)\left(\frac{1}{2}+\frac{\gamma}{2}-\varepsilon\right)
$$

Now the claim of the lemma follows if we can ensure that $\frac{2}{3} \alpha>\frac{1}{3}$. A direct computation shows that the last inequality is equivalent to $\gamma>4 / 3+10 \varepsilon / 3$, which can be assured for $\varepsilon$ small enough, thanks to our assumption that $\gamma>\frac{4}{3}$. This completes the proof of Lemma 5.3

Summarizing the previous discussion provides the required bound for $I_{1}$. This completes the proof of Proposition 5.1. 
REMARK 5.4. Let us observe that for $\gamma>2$ one may obtain the analogue of Proposition 5.1 by using an argument which does not require the integration by parts trick on the quantity (5.1). Indeed, for $\gamma>2$, the expression

$$
\int\left(\left(1+\left|D_{x}\right|^{\gamma}\right)^{-1}\left|D_{x}\right|^{s+\gamma} \pi_{N} u\right)\left|D_{x}\right|^{s}\left(\partial_{x}\left(\pi_{N} u\right)^{2}\right)
$$

has enough smoothing that we can employ a semilinear technique to achieve the desired bound. Interestingly, $\gamma=2$ is also the border line of the applicability of the result of [32].

\section{A large-deviation bound}

We can now invoke the following large-deviation estimate for the quantity appearing in the energy estimate.

Lemma 6.1. Let $\varepsilon>0$. There exists $C$ such that, for every $r>0$, every $p \geqslant 2$, and every $N \geqslant 1$,

$$
\|\|\left|D_{x}\right|^{s+\gamma / 2-1 / 2-\varepsilon} \pi_{N} u\left\|_{L^{\infty}}\right\|_{L^{p}\left(\mu_{s, r}(u)\right)} \leqslant C p^{1 / 2} .
$$

Proof. We first observe that

$$
\|\|\left|D_{x}\right|^{s+\gamma / 2-1 / 2-\varepsilon} \pi_{N} u\left\|_{L^{\infty}}\right\|_{L^{p}\left(\mu_{s, r}(u)\right)} \leqslant\|\|\left|D_{x}\right|^{s+\gamma / 2-1 / 2-\varepsilon} \pi_{N} u\left\|_{L^{\infty}}\right\|_{L^{p}\left(\mu_{s}(u)\right)} .
$$

Therefore, we need to prove that

$$
\|\|\left|D_{x}\right|^{s+\gamma / 2-1 / 2-\varepsilon} \pi_{N} u\left\|_{L^{\infty}}\right\|_{L^{p}\left(\mu_{s}(u)\right)} \leqslant C p^{1 / 2} .
$$

Coming back to the definition of $\mu_{s}$, the last inequality can be rewritten as

$$
\|\|\left|D_{x}\right|^{s+\gamma / 2-1 / 2-\varepsilon} \sum_{n \neq 0,|n| \leqslant N} \frac{g_{n}(\omega)}{|n|^{s+\gamma / 2}} e^{i n x}\left\|_{L^{\infty}}\right\|_{L_{\omega}^{p}} \leqslant C p^{1 / 2},
$$

which would follow from

$$
\|\| \sum_{n \neq 0,|n| \leqslant N} \frac{g_{n}(\omega)}{|n|^{1 / 2+\varepsilon}} e^{i n x}\left\|_{L^{\infty}}\right\|_{L_{\omega}^{p}} \leqslant C p^{1 / 2} .
$$

Now, using the Sobolev embedding, we obtain that the last inequality follows from

$$
\|\| \sum_{n \neq 0,|n| \leqslant N} \frac{g_{n}(\omega)}{|n|^{1 / 2+\varepsilon / 2}} e^{i n x}\left\|_{L^{q}}\right\|_{L_{\omega}^{p}} \leqslant C p^{1 / 2},
$$

provided that $q>2 / \varepsilon$. The inequality (6.1) is classical (see, for example, [14, Lemma 3.1]). This completes the proof of Lemma 6.1. 


\section{The measure evolution property}

LEMMA 7.1. There is $0 \leqslant \beta<1$ such that for every $r>0$ there is a constant $C>0$ such that, for every $p \geqslant 2$ and every Borel set $A$ of $H^{s}$, and for every $N \geqslant 1$,

$$
\frac{d}{d t} \mu_{s, r}\left(\Phi_{N}(t)(A)\right) \leqslant C p^{\beta}\left(\mu_{s, r}\left(\Phi_{N}(t)(A)\right)\right)^{1-1 / p} .
$$

REMARK 7.2. We remark that, in a similar situation in [36], the quantity

$$
\left(\mu_{s, r}\left(\Phi_{N}(t)(A)\right)\right)^{1-1 / p}
$$

is simply bounded by one. Thanks to the use of much more subtle energies related to the remarkable but very particular structure of the Benjamin-Ono equation, in [36] the contribution corresponding to $C p^{\beta}$ is a (delicate) quantity tending to zero as $N \rightarrow \infty$ (which leads to the invariance of the corresponding measure, while here we only get quasiinvariance).

Proof of Lemma 7.1. Using the flow properties of $\Phi_{N}(t)$, we obtain that

$$
\begin{aligned}
\left.\frac{d}{d t} \mu_{s, r}\left(\Phi_{N}(t)(A)\right)\right|_{t=\bar{t}} & =\left.\frac{d}{d t} \int_{\Phi_{N}(t)(A)} \chi_{r}(u) d \mu_{s, r}(u)\right|_{t=\bar{t}} \\
& =\left.\frac{d}{d t} \int_{\Phi_{N}(t)\left(\Phi_{N}(\bar{t})(A)\right)} \chi_{r}(u) d \mu_{s, r}(u)\right|_{t=0} \equiv I .
\end{aligned}
$$

Using Proposition 4.1, we can write $I$ as

$$
I=\left.\gamma_{N} \frac{d}{d t} \int_{\Phi_{N}(\bar{t})(A)} \chi_{r}(u) e^{-\left\|\pi_{N}\left(\Phi_{N}(t)(u)\right)\right\|_{H^{s+\gamma / 2}}^{2}} d u_{1} \ldots d u_{N} d \mu_{s ; N}^{\perp}\right|_{t=0} .
$$

Therefore

$$
\begin{aligned}
I= & -\gamma_{N} \int_{\Phi_{N}(\bar{t})(A)} \chi_{r}(u)\left(\left.\frac{d}{d t}\left\|\pi_{N}\left(\Phi_{N}(t)(u)\right)\right\|_{H^{s+\gamma / 2}}^{2}\right|_{t=0}\right) \\
& \times e^{-\left\|\pi_{N}(u)\right\|_{H^{s+\gamma / 2}}^{2}} d u_{1} \ldots d u_{N} d \mu_{s ; N}^{\perp},
\end{aligned}
$$

and consequently

$$
I=-\int_{\Phi_{N}(\bar{t})(A)}\left(\left.\frac{d}{d t}\left\|\pi_{N}\left(\Phi_{N}(t)(u)\right)\right\|_{H^{s+\gamma / 2}}^{2}\right|_{t=0}\right) d \mu_{s, r}(u) .
$$

Therefore, using the Hölder inequality, we can write

$$
I \leqslant\left\|\frac{d}{d t}\right\| \pi_{N}\left(\Phi_{N}(t)(u)\right)\left\|\left._{H^{s+\gamma / 2}}^{2}\right|_{t=0}\right\|_{L^{p}\left(\mu_{s, r}(u)\right)}\left(\mu_{s, r}\left(\Phi_{N}(\bar{t})(A)\right)\right)^{1-1 / p} .
$$


Therefore, it remains to show that

$$
\left\|\frac{d}{d t}\right\| \pi_{N}\left(\Phi_{N}(t)(u)\right)\left\|\left._{H^{s+\gamma / 2}}^{2}\right|_{t=0}\right\|_{L^{p}\left(\mu_{s, r}(u)\right)} \leqslant C p^{\beta},
$$

for some $\beta<1$. At this point, we shall invoke the energy estimate of Proposition 5.1. Namely, using Proposition 5.1, we can write

$$
\begin{aligned}
\| \frac{d}{d t} & \left.\left\|\pi_{N}\left(\Phi_{N}(t)(u)\right)\right\|_{s+\gamma / 2}^{2}\right|_{t=0} \|_{L^{p}\left(\mu_{s, r}(u)\right)} \\
\leqslant & C \|\left(1+\left\|\pi_{N} \Phi_{N}(t)(u)\right\|_{H^{\gamma / 2}}^{3-\kappa}\right) \\
& \times\left.\left(1+\left\|\left|D_{x}\right|^{s+\gamma / 2-1 / 2-\varepsilon} \pi_{N} \Phi_{N}(t)(u)\right\|_{L^{\infty}}^{\kappa}\right)\right|_{t=0} \|_{L^{p}\left(\mu_{s, r}(u)\right)} \\
\leqslant & C\left(1+r^{3-\kappa}\right)\left(1+\|\|\left|D_{x}\right|^{s+\gamma / 2-1 / 2-\varepsilon} \pi_{N} u\left\|_{L^{\infty}}\right\|_{L^{\kappa p}\left(\mu_{s, r}(u)\right)}^{\kappa}\right),
\end{aligned}
$$

for some $\kappa<2$.

Using Lemma 6.1 and (7.1), we obtain that

$$
\left\|\frac{d}{d t}\right\| \pi_{N}\left(\Phi_{N}(t)(u)\right)\left\|\left._{H^{s+\gamma / 2}}^{2}\right|_{t=0}\right\|_{L^{p}\left(\mu_{s, r}(u)\right)} \leqslant C p^{\kappa / 2} .
$$

This completes the proof of Lemma 7.1.

We are now in a position to apply a variant of the Yudovich argument [39].

LEMMA 7.3. Fix $t \in \mathbb{R}, r \geqslant 0$, and $\delta>0$. There exists $C>0$ such that, for every Borel set $A$ of $H^{s}$, and for every $N \geqslant 1, \mu_{s, r}\left(\Phi_{N}(t)(A)\right) \leqslant C\left(\mu_{s, r}(A)\right)^{1-\delta}$.

Proof. The conclusion of Lemma 7.1 can be written as

$$
\frac{d}{d t}\left(\mu_{s, r}\left(\Phi_{N}(t)(A)\right)\right)^{1 / p} \leqslant C p^{-\alpha},
$$

where $\alpha=1-\beta>0$. After an integration, we obtain that

$$
\begin{aligned}
\mu_{s, r}\left(\Phi_{N}(t)(A)\right) & \leqslant\left(\left(\mu_{s, r}(A)\right)^{1 / p}+C t p^{-\alpha}\right)^{p} \\
& =\mu_{s, r}(A) e^{p \log \left(1+C t p^{-\alpha}\left(\mu_{s, r}(A)\right)^{-1 / p}\right)} .
\end{aligned}
$$

Using that, for $x \geqslant 0$, one has $\log (1+x) \leqslant x$, we arrive at the bound

$$
\mu_{s, r}\left(\Phi_{N}(t)(A)\right) \leqslant \mu_{s, r}(A) e^{C t p^{1-\alpha}\left(\mu_{s, r}(A)\right)^{-1 / p}} .
$$

We now choose $p$ as

$$
p \equiv 2+\log \left(\frac{1}{\mu_{s, r}(A)}\right)
$$


Therefore we obtain that

$$
\mu_{s, r}\left(\Phi_{N}(t)(A)\right) \leqslant \mu_{s, r}(A) e^{\operatorname{Cet}\left(2+\log \left(\frac{1}{\mu s, r(A)}\right)\right)^{1-\alpha}} .
$$

We therefore conclude that for every $\delta>0$ there is a constant $\tilde{C}=\tilde{C}(\delta, \alpha, C, t)$ (that is, depending also on $\alpha, C$, and $t$ ) such that

$$
\mu_{s, r}\left(\Phi_{N}(t)(A)\right) \leqslant \tilde{C}(\delta, \alpha, C, t)\left(\mu_{s, r}(A)\right)^{1-\delta} .
$$

This completes the proof of Lemma 7.3.

REMARK 7.4. Using the argument of [7], the bound (7.2) may be used to obtain that the $H^{s+\gamma / 2-1 / 2-\varepsilon}$-norms of the solutions with data on the support of $\mu_{s}$ do not grow faster than a quantity of type $t^{\gamma(s)}(t \gg 1)$ with $\gamma(s) \rightarrow \infty$ as $s \rightarrow \infty(\gamma(s)$ may be taken close to $s-\frac{1}{2}$ ). However, such a bound may be achieved by purely deterministic methods. On the other hand, if the bound (7.2) is replaced by the stronger bound

$$
\mu_{s, r}\left(\Phi_{N}(t)(A)\right) \leqslant \mu_{s, r}(A) e^{C t},
$$

then the argument of [7] would give a bound of type $t^{1 / 2}$. Such a bound would be of greater interest, because the power is independent of $s$.

\section{End of the proof of the main result}

LEMMA 8.1. Fix $t \in \mathbb{R}, r>0, R>0$, and $\delta>0$. There exists $C>0$ such that, for every Borel set $A \subset B_{R, s}$ of $H^{s}, \mu_{s, r}(\Phi(t)(A)) \leqslant C\left(\mu_{s, r}(A)\right)^{1-\delta}$.

Proof. We first show the statement of Lemma 8.1 if $A \subset B_{R, s}$ is a compact set. In this case, using Propositions 2.10 and 7.3, we obtain that, for every $\varepsilon>0$, there is $N_{0}$ such that, for every $N \geqslant N_{0}$,

$$
\mu_{s, r}(\Phi(t)(A)) \leqslant \mu_{s, r}\left(\Phi_{N}(t)\left(A+B_{\varepsilon, s}\right)\right) \leqslant C(\delta, t, r)\left(\mu_{s, r}\left(A+B_{\varepsilon, s}\right)\right)^{1-\delta} .
$$

Now, since $A$ is a compact, using the dominate convergence theorem, we obtain that, in the limit $\varepsilon \rightarrow 0$,

$$
\mu_{s, r}(\Phi(t)(A)) \leqslant C(\delta, t, r)\left(\mu_{s, r}(A)\right)^{1-\delta} .
$$

Let now $A \subset B_{R, s}$ be an arbitrary Borel set. Using the regularity of $\mu_{s, r}$, we obtain that there is a sequence $\left(K_{n}\right)_{n=1}^{\infty}$ of compacts of $H^{s}$ such that $K_{n} \subset \Phi(t)(A)$ and

$$
\lim _{n \rightarrow \infty} \mu_{s, r}\left(K_{n}\right)=\mu_{s, r}(\Phi(t)(A)) .
$$


We next observe that

$$
K_{n} \subset \Phi(t)\left(\Phi(-t)\left(K_{n}\right)\right)
$$

Indeed, every $x \in K_{n}$ can be written as

$$
x=\Phi(t)(\Phi(-t)(x)) \in \Phi(t)\left(\Phi(-t)\left(K_{n}\right)\right) .
$$

Thus we have (8.2). Using (8.2), we infer that

$$
\mu_{s, r}\left(K_{n}\right) \leqslant \mu_{s, r}\left(\Phi(t)\left(F_{n}\right)\right), F_{n}=\Phi(-t)\left(K_{n}\right) .
$$

Since $K_{n}$ is a compact and $\Phi(-t)$ is a continuous map, we obtain that $F_{n}$ is a compact. We now claim that

$$
F_{n} \subset A \text {. }
$$

Indeed, let $x \in F_{n}$. This means that there is $y \in K_{n}$ such that $x=\Phi(-t)(y)$. But $K_{n} \subset \Phi(t)(A)$, and therefore $y \in \Phi(t)(A)$. As a consequence, there exists $z \in A$ such that $y=\Phi(t)(z)$. Since $x=\Phi(-t)(y)$ and $y=\Phi(t)(z)$, we infer that $x=z$. Therefore $x \in A$, and the proof of (8.4) is complete. Thanks to the analysis for compact sets performed at the beginning of the proof, we obtain that

$$
\mu_{s, r}\left(\Phi(t)\left(F_{n}\right)\right) \leqslant C(\delta, t, r)\left(\mu_{s, r}\left(F_{n}\right)\right)^{1-\delta} \leqslant C(\delta, t, r)\left(\mu_{s, r}(A)\right)^{1-\delta} .
$$

Coming back to (8.3), we obtain the bound

$$
\mu_{s, r}\left(K_{n}\right) \leqslant C(\delta, t, r)\left(\mu_{s, r}(A)\right)^{1-\delta} .
$$

Passing to the limit $n \rightarrow \infty$ by invoking (8.1) gives

$$
\mu_{s, r}(\Phi(t)(A)) \leqslant C(\delta, t, r)\left(\mu_{s, r}(A)\right)^{1-\delta} .
$$

This completes the proof of Lemma 8.1.

Let us now complete the proof of Theorem 1.2. Let $A$ be a Borel set of $H^{s}$ such that $\mu_{s}(A)=0$. Our goal is to show that $\mu_{s}(\Phi(t)(A))=0$. Since $\mu_{s}(A)=0$, we also have that ,for every $R, r>0, \mu_{s, r}\left(A \cap B_{R, s}\right)=0$. Therefore, thanks to Lemma 8.1, for every $R, r>0, \mu_{s, r}\left(\Phi(t)\left(A \cap B_{R, s}\right)\right)=0$. On the other hand, thanks to the dominated convergence theorem, for every Borel set $A$ of $H^{s}$,

$$
\mu_{s}(A)=\lim _{r \rightarrow \infty} \mu_{s, r}(A) .
$$

This implies that, for every $R>0, \mu_{s}\left(\Phi(t)\left(A \cap B_{R, s}\right)\right)=0$. Now, we invoke the straightforward property

$$
\left.\Phi(t)(A)=\bigcup_{R=1}^{\infty} \Phi(t)\left(A \cap B_{R, s}\right)\right)
$$

to obtain that $\mu_{s}(\Phi(t)(A))=0$. This completes the proof of Theorem 1.2. 


\section{Proof of Proposition 1.3}

We have that $\Sigma(t)(u)=S(t)(u)+f(t)$, where $f(t)$ is given by

$$
f(t) \equiv-\int_{0}^{t} S(t-\tau)\left(\left(1+\left|D_{x}\right|^{\gamma}\right)^{-1} \partial_{x}(h)\right) d \tau .
$$

We have that $f(t) \in H^{\sigma}$, thanks to the hypothesis on $h$. Since $\mu_{s}$ is invariant under $S(t)$, we have that, for every $g \in L^{1}\left(d \mu_{s}(u)\right)$,

$$
\int_{H^{s}} g(S(t)(u)+f(t)) d \mu_{s}(u)=\int_{H^{s}} g(u+f(t)) d \mu_{s}(u),
$$

and therefore we need to show that the image measure of $\mu_{s}$ under the map $u \mapsto$ $u+f(t)$ (seen as a bijection on $H^{\sigma}$ ) is singular with respect to $\mu_{s}$. We now show that

$$
f(t) \notin H^{s+\gamma / 2}, \quad t \neq 0 .
$$

We have

$$
S(t-\tau)\left(\left(1+\left|D_{x}\right|^{\gamma}\right)^{-1} \partial_{x}(h)\right)=\sum_{n} \frac{i n}{1+|n|^{\gamma}} \hat{h}(n) e^{i n x} e^{-i(t-\tau) n /\left(1+|n|^{\gamma}\right)},
$$

and using that for $t \neq 0$ there is $c>0$ such that, for every $n$ large enough,

$$
\left|\int_{0}^{t} e^{-i(t-\tau) n /\left(1+|n|^{\gamma}\right)} d \tau\right| \geqslant c
$$

we obtain that, for $\gamma<\frac{3}{2}$,

$$
\|f(t)\|_{H^{s+\gamma / 2}} \geqslant c\|h\|_{H^{s-\gamma / 2+1}} \geqslant c\|h\|_{H^{s+\gamma / 2-1 / 2}}=+\infty .
$$

This completes the proof of (9.1). Therefore we can apply the Cameron-Martin argument, as we now explain. Thanks to (9.1), for every $t \neq 0$ there is $k \in H^{s+\gamma / 2}$ such that

$$
\sum_{n \neq 0}|n|^{2(s+\gamma / 2)} \widehat{f(t)}(n) \overline{\hat{k}(n)}=+\infty .
$$

The existence of $k$ may be obtained either by invoking the Banach-Steinhaus theorem or by an explicit construction. Next, we observe that

$$
\mu_{s}\left(u: \sum_{n \neq 0}|n|^{2(s+\gamma / 2)} \hat{u}(n) \overline{\hat{k}(n)}<\infty\right)=p\left(\omega: \sum_{n \neq 0}|n|^{s+\gamma / 2} g_{n}(\omega) \overline{\hat{k}(n)}<\infty\right)=1,
$$


where we used that the basic orthogonality between $\left(g_{n}\right)$ yields

$$
\left\|\sum_{n \neq 0}|n|^{s+\gamma / 2} g_{n}(\omega) \overline{\hat{k}(n)}\right\|_{L_{\omega}^{2}} \lesssim\|k\|_{H^{s+\gamma / 2}} .
$$

Thanks to (9.3), there is a set $A \subset H^{s}$ such that $\mu_{s}(A)=1$ and, for every $v \in A$,

$$
\sum_{n \neq 0}|n|^{2(s+\gamma / 2)} \hat{v}(n) \overline{\hat{k}(n)}<\infty .
$$

Let us denote by $\mu_{s}^{t}$ the image measure of $\mu_{s}$ under the map $u \mapsto u+f(t)$. Then

$$
\mu_{s}^{t}(A)=\mu_{s}(B), \quad B \equiv\{v-f(t), v \in A\} .
$$

Thanks to (9.2) and (9.4), we obtain that, for every $u \in B$,

$$
\sum_{n \neq 0}|n|^{2(s+\gamma / 2)} \hat{u}(n) \overline{\hat{k}(n)}=\infty .
$$

Therefore $B \subset A^{c}$, and consequently $\mu_{s}(B)=0$; that is, $\mu_{s}^{t}(A)=0$. Since $\mu_{s}(A)=1$, we conclude that $\mu_{s}$ and $\mu_{s}^{t}$ are mutually singular. This completes the proof of Proposition 1.3.

\section{Final remarks}

The arguments we presented here can be seen as a combination of the use of higher-order pseudoenergies and the idea of [36] reducing the analysis of the transported measure to a property of the random series describing the set of the initial data. In this work we presented this approach in the simplest significant setting we found, namely the generalized BBM models. It would be interested to decide how much the results obtained here can be extended to other Hamiltonian PDEs. For instance, we believe that a slight modification of the proof of Theorem 1.2 gives the quasiinvariance of the Gaussian measures $\mu_{s}$ under the flow of the one-dimensional Klein-Gordon equation

$$
\partial_{t}^{2} u-\partial_{x}^{2} u+u+u^{3}=0 .
$$

Such a result would however be at the border line of the Ramer result (there is 1-derivative smoothing when rewriting (10.1) as a first-order equation) and moreover it does not go beyond the Cameron-Martin threshold. Consequently, we find it less interesting than Theorem 1.2. The extension to two dimensions in the context of (10.1) is an interesting issue, which is not part of our present 
understanding of this set of problems. Another issue which may be interesting is whether one may incorporate a dispersive effect in measure quasiinvariance problems; that is, whether one may exploit more subtle smoothing properties related to dispersion (see, for example, [2, 6, 24]). Finally, it would be very interesting to find situations where we can prove that the transported measure is singular with respect to the initial Gaussian measure, and describe the measure evolution.

\section{Acknowledgements}

I am indebted to Nicolas Burq and Nicola Visciglia, since this work benefited from our collaborations on related topics. I am grateful to Ana Bela Cruzeiro, Huang Guan, and Tadahiro Oh for discussions on the subject discussed in this paper. This work was supported by the European Research Council (grant no. 257293 Dispeq). I am grateful to the referees for their remarks and suggestions.

\section{References}

[1] L. Ambrosio and A. Figalli, 'On flows associated to Sobolev vector fields in Wiener spaces: an approach a la DiPerna-Lions', J. Funct. Anal. 256 (2009), 179-214.

[2] A. Babin, A. Ilyin and E. Titi, 'On the regularization mechanism for the periodic Kortewegde Vries equation', Comm. Pure Appl. Math. 64 (2011), 591-648.

[3] B. Benjamin, J. Bona and J. Mahony, 'Model equations for long waves in nonlinear dispersive systems', Philos. Trans. R. Soc. Lond. Ser. A Math. Phys. Sci. 272 (1972), 47-78.

[4] V. Bogachev, Gaussian Measures, Mathematical Surveys and Monographs, 62 (American Mathematical Society, Providence, RI, 1998).

[5] V. Bogachev and E. Mayer-Wolf, 'Absolutely continuous flows generated by Sobolev class vector fields in finite and infinite dimensions', J. Funct. Anal. 167 (1999), 1-68.

[6] J. Bourgain, 'Fourier transform restriction phenomena for certain lattice subsets and applications to nonlinear evolution equations, Part II', GAFA 3 (1993), 209-262.

[7] J. Bourgain, 'Periodic nonlinear Schrödinger equation and invariant measures', Comm. Math. Phys. 166 (1994), 1-26.

[8] J. Bourgain, 'Invariant measures for the 2d-defocusing nonlinear Schrödinger equation', Comm. Math. Phys. 176 (1996), 421-445.

[9] J. Bourgain and A. Bulut, 'Invariant Gibbs measure evolution for the radial nonlinear wave equation on the 3D ball', J. Funct. Anal. 266 (2014), 2319-2340.

[10] J. Bourgain and A. Bulut, 'Almost sure global well posedness for the radial nonlinear Schrodinger equation on the unit ball I: the 2D case', Ann. Inst. H. Poincaré Anal. Non Linéaire 31 (2013), 1267-1288.

[11] J. Bourgain and A. Bulut, 'Almost sure global well posedness for the radial nonlinear Schrodinger equation on the unit ball II: the 3D case', J. Eur. Math. Soc. 16 (2014), 1289-1325.

[12] N. Burq, P. Gérard and N. Tzvetkov, 'Strichartz inequalities and the nonlinear Schrödinger equation on compact manifolds', Amer. J. Math. 126 (2004), 569-605. 
[13] N. Burq and N. Tzvetkov, 'Invariant measure for a three dimensional nonlinear wave equation', Int. Math. Res. Not. IMRN (2007), 1-26.

[14] N. Burq and N. Tzvetkov, 'Random data Cauchy theory for supercritical wave equations I. Local theory', Invent. Math. 173 (2008), 449-475.

[15] N. Burq and N. Tzvetkov, 'Random data Cauchy theory for supercritical wave equations II. A global existence result', Invent. Math. 173 (2008), 477-496.

[16] N. Burq, L. Thomann and N. Tzvetkov, 'Long time dynamics for the one dimensional non linear Schrödinger equation', Ann. Inst. Fourier 63 (2013), 2137-2198.

[17] R. H. Cameron and W. T. Martin, 'Transformation of Wiener integrals under translations', Ann. of Math. (2) 45 (1944), 386-396.

[18] A. B. Cruzeiro, 'Equations différentielles ordinaire: non explosition et mesures quasiinvariantes', J. Funct. Anal. 54 (1983), 193-206.

[19] A. B. Cruzeiro, 'Equations différentielles sur l'espace de Wiener et formules de CameronMartin non linéaires', J. Funct. Anal. 54 (1983), 206-227.

[20] Y. Deng, 'Two dimensional NLS equation with random radial data', Anal. PDE 5 (2012), 913-960.

[21] Y. Deng, 'Invariance of the Gibbs measure for the Benjamin-Ono equation', J. Eur. Math. Soc. 17 (2015), 1107-1198.

[22] A. S. de Suzzoni, 'Wave turbulence for the BBM equation: stability of a Gaussian statistics under the flow of BBM', Comm. Math. Phys. 326 (2014), 773-813.

[23] A. S. de Suzzoni, 'Invariant mesure for the cubic non linear wave equation on the unit ball of $R^{3}$, Dyn. Partial Differ. Equ. 8 (2011), 127-147.

[24] M. Erdogan and N. Tzirakis, 'Global smoothing for the periodic KdV evolution', Int. Math. Res. Not. IMRN (2012), 4589-4614.

[25] D. Lannes, The Water Waves Problem: Mathematical Analysis and Asymptotics, Mathematical Surveys and Monographs (American Mathematical Society, 2013).

[26] J. Lebowitz, R. Rose and E. Speer, 'Statistical dynamics of the nonlinear Schrödinger equation', J. Stat. Phys. V 50 (1988), 657-687.

[27] A. Nahmod, T. Oh, L. Rey-Bellet and G. Staffilani, 'Invariant weighted Wiener measures and almost sure global well-posedness for the periodic derivative NLS', J. Eur. Math. Soc. 14 (2012), 1275-1330.

[28] A. Nahmod, L. Rey-Bellet, S. Sheffield and G. Staffilani, 'Absolute continuity of Brownian bridges under certain gauge transformations', Math. Res. Lett. 18(5) (2011), 875-887.

[29] T. Oh, 'Invariance of the Gibbs measure for the Schrödinger-Benjamin-Ono system', SIAM J. Math. Anal. 41 (2009), 2207-2225.

[30] T. Oh, 'Invariance of the white noise for KdV', Comm. Math. Phys. 292(1) (2009), 217-236.

[31] P. J. Olver, 'Euler operators and conservation laws of the BBM equation', Math. Proc. Cambridge Philos. Soc. 85 (1979), 143-160.

[32] R. Ramer, 'On nonlinear transformations of Gaussian measures', J. Funct. Anal. 15 (1974), 166-187.

[33] G. Richards, 'Invariance of the Gibbs measure for the periodic quartic gKdV', Ann. Inst. H. Poincaré Anal. Non Linéaire, to appear.

[34] L. Thomann and N. Tzvetkov, 'Gibbs measure for the periodic derivative non linear Schrödinger equation', Nonlinearity 23 (2010), 2771-2791.

[35] N. Tzvetkov, 'Invariant measures for the defocusing NLS', Ann. Inst. Fourier 58 (2008), 2543-2604.

[36] N. Tzvetkov and N. Visciglia, 'Invariant measures and long-time behavior for the BenjaminOno equation', Int. Math. Res. Not. IMRN (2014), 4679-4714. 
[37] N. Tzvetkov and N. Visciglia, 'Invariant measures and long time behaviour for the Benjamin-Ono equation II', J. Math. Pures Appl. 103 (2015), 102-141.

[38] J. Quastel and B. Valko, 'KdV preserves white noise', Comm. Math. Phys. 277 (2008), 707-714.

[39] V. Yudovich, 'Non-stationary flows of an ideal incompressible fluid', Zh. Vychisl. Mat. Mat. Fiz. (1963), 1032-1066. (in Russian).

[40] P. Zhidkov, KdV and Nonlinear Schrödinger Equations: Qualitative Theory, Lecture Notes in Mathematics, 1756 (Springer, 2001). 